\title{
2,3-DIHYDROIMIDAZO[1,2-A]PYRIDINES: A NEW CLASS OF ENANTIOSELECTIVE ACYL TRANSFER CATALYSTS AND THEIR USE IN KINETIC RESOLUTION OF ALCOHOLS
}

\author{
Vladimir B. Birman, * Eric W. Uffman and Corey J. Kilbane \\ A contribution from the Department of Chemistry, Washington University, Campus Box 1134, \\ One Brookings Drive, Saint Louis, Missouri 63130
}

SUPPORTING INFORMATION: Experimental procedures (11 pages), ${ }^{1} \mathrm{H}$ NMR and ${ }^{13} \mathrm{C}$ NMR spectra (20 pages).

\section{Experimental Part.}

\begin{abstract}
A) General. All reagents used for the preparation of the catalysts were obtained commercially and were used as received. Reactions involving higher than atmospheric pressures were carried out in $25 \mathrm{~mL}$ Cajon ${ }^{\circledR}$ Airfree ${ }^{\circledR}$ storage vessels available from Chemglass. The substrates used in the kinetic resolution experiments were either purchased or prepared (either by borohydride reduction of the corresponding ketones or by reacting aromatic aldehydes with Grignard reagents) and were purified as necessary. Chloroform (OmniSolv® grade, stabilized with nonpolar hydrocarbons) was used as received from EM Science. Deuterated chloroform was used as received from Cambridge Isotope Laboratories, Inc. Solvents used for chromatography were ACS or HPLC grade, as appropriate. Reactions were monitored by thin layer chromatography (TLC) using EM Science 60F silica gel plates. Flash column chromatography was performed over ICN Ecochrom silica gel (32-63 $\square \mathrm{m})$. Melting points were measured on a Mel-Temp II capillary melting point apparatus and are uncorrected. ${ }^{1} \mathrm{H}$ NMR and ${ }^{13} \mathrm{C}$ NMR spectra were recorded on a Unity $300 \mathrm{MHz}$ Varian spectrometer. The chemical shifts are reported as $\square$ values (ppm) relative to TMS. High-Resolution mass spectral analyses were performed at Washington University MS Center on a Kratos MS-50TA spectrometer using the fast atom bombardment method (FAB). Methods used for kinetic resolution experiments, determination of ee's and calculation of conversions and selectivities were adopted from previously published work. ${ }^{3 b, 4 a}$ HPLC analyses were performed using a Breeze LC system (Waters Corporation) using isopropanol-hexanes mobile phase at a flow rate of $1 \mathrm{~mL} / \mathrm{min}$ and UV detection at $254 \mathrm{~nm}$. Specific optical rotations were measured using a Perkin-Elmer 241 polarimeter. The absolute chirality of the esters and alcohols obtained by kinetic resolution was determined by the sign of the optical rotation of the unreacted alcohol.
\end{abstract}




\section{B) Preparation of the catalysts}

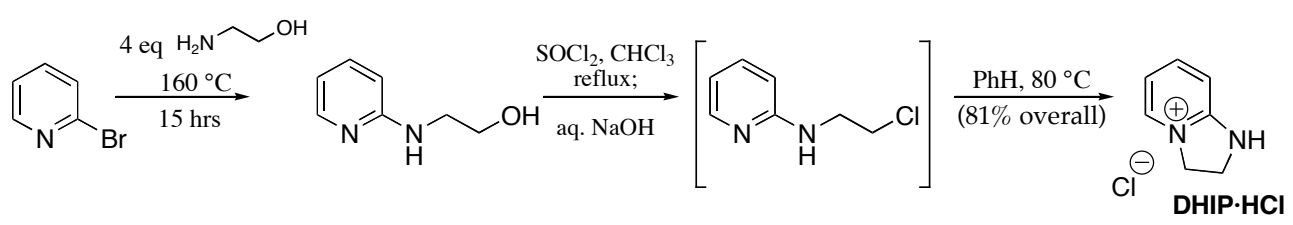

\section{2, 3-Dihydroimidazo[1,2-a]pyridine hydrochloride}

A combination of several existing procedures was adapted for the preparation of DHIP hydrochloride ${ }^{\text {atc }}$ The following protocol was used only once and is unoptimized. A mixture of 2-bromopyridine (3.16 g, $20.0 \mathrm{mmol})$ and ethanolamine (4.88 g, $80.0 \mathrm{mmol})$ was heated at 160 ${ }^{\circ} \mathrm{C}$ under reflux in an oil bath for 15 hours. The reflux condenser was replaced with a distillation condenser and most of the unreacted ethanolamine was removed under reduced pressure (bath temperature $\left.150{ }^{\circ} \mathrm{C}\right)$. The remaining oil $(5.37 \mathrm{~g})$ was triturated with $\mathrm{CH}_{2} \mathrm{Cl}_{2}$ and left to crystallize. The crystalline ethanolamine hydrobromide was filtered off and rinsed with $\mathrm{CH}_{2} \mathrm{Cl}_{2}$ on the filter. The filtrate was evaporated to give $3.34 \mathrm{~g}$ of yellow oil. The oil was diluted with $20 \mathrm{~mL}$ of chloroform, cooled to $0^{\circ} \mathrm{C}$ and treated with thionyl chloride $(3.0 \mathrm{~mL}, 41 \mathrm{mmol})$ added cautiously dropwise. The resulting solution was heated under reflux (bath temperature $75{ }^{\circ} \mathrm{C}$ ) for 1 hour. The solvent was evaporated, and the residue was treated with $1 \mathrm{~mL}$ of $\mathrm{MeOH}$. The resulting beige, crystalline mass was dissolved in $15 \mathrm{~mL}$ of water and $25 \mathrm{~mL}$ of benzene was added. The two-phase mixture was cooled in an ice bath and then basified with aqueous $\mathrm{NaOH}$ $\left(2 \mathrm{~g} / 5 \mathrm{~mL} \mathrm{H}_{2} \mathrm{O}\right.$ ) with vigorous magnetic stirring. The organic layer was separated and the aqueous layer was extracted twice with benzene $(2 \times 25 \mathrm{~mL})$. The combined organic extract was washed with brine, dried over $\mathrm{Na}_{2} \mathrm{SO}_{4}$ and kept at room temperature for 1.5 hours. The lightyellow solution was decanted off the dark-brown oil that had precipitated and heated under reflux (bath temperature $85^{\circ} \mathrm{C}$ ). Light-beige crystals precipitated slowly. After two hours, the reaction was complete by TLC. The precipitate was filtered off and dried over Drierite ${ }^{\circledR}$ overnight to give $2.535 \mathrm{~g}$ of the crude product ( $81 \%$ yield) which was sufficiently pure for subsequent experiments.

${ }^{1} \mathbf{H}$ NMR $\left(300 \mathrm{MHz}, \mathrm{D}_{2} \mathrm{O}, \mathrm{HOD}\right.$ peak at $\square 4.80 \mathrm{ppm}$ used as reference) $\square 7.97(\mathrm{~d} ; \mathrm{J}=6.6 \mathrm{~Hz}$, $1 \mathrm{H}$ ), 7.88 (ddd; $\left.\mathrm{J}_{1}=8.8 \mathrm{~Hz}, \mathrm{~J}_{2}=7.4 \mathrm{~Hz}, \mathrm{~J}_{3}=1.4 \mathrm{~Hz}, 1 \mathrm{H}\right), 7.00(\mathrm{~d} ; \mathrm{J}=9.0 \mathrm{~Hz}, 1 \mathrm{H}), 6.89$ (ddd; $\mathrm{J}_{1}$ $\left.=7.4 \mathrm{~Hz}, \mathrm{~J}_{2}=7.1 \mathrm{~Hz}, \mathrm{~J}_{3}=1.1 \mathrm{~Hz}, 1 \mathrm{H}\right), 4.70\left(\mathrm{dd} ; \mathrm{J}_{1}=10.1 \mathrm{~Hz}, \mathrm{~J}_{2}=9.6 \mathrm{~Hz}, 2 \mathrm{H}\right), 4.01\left(\mathrm{dd} ; \mathrm{J}_{1}=\right.$ $\left.10.1 \mathrm{hz}, \mathrm{J}_{2}=9.6 \mathrm{~Hz}, 2 \mathrm{H}\right) ;{ }^{13} \mathbf{C ~ N M R}\left(75 \mathrm{MHz}, \mathrm{D}_{2} \mathrm{O}, \mathrm{CH}_{3} \mathrm{OH}\right.$ added and used as reference peak at $\square 49.50 \mathrm{ppm}) \square 145.1,137.0,114.3,110.0,51.6,43.3$ (quaternary carbon C7a not observed).

\section{2, 3-Dihydroimidazo[1,2-a]pyridine base}

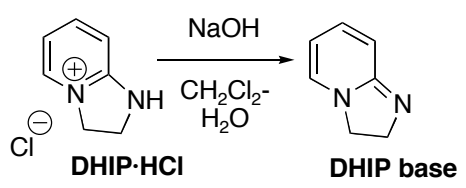

The hydrochloride obtained as described above $(156 \mathrm{mg}, 1.00 \mathrm{mmol})$ was dissolved in $1 \mathrm{~mL}$ of water and treated with $2 \mathrm{~mL}$ of $33 \%$ aqueous $\mathrm{NaOH}$. The dark-yellow oil that separated out 
was extracted with $\mathrm{CH}_{2} \mathrm{Cl}_{2}$ several times until the aqueous phase became pale-yellow. The organic extract was dried over $\mathrm{NaOH}$ pellets and rotary evaporated leaving $121 \mathrm{mg}$ of oil (quantitative yield), which was dissolved in $\mathrm{CDCl}_{3}$, evaporated again to remove traces of $\mathrm{CH}_{2} \mathrm{Cl}_{2}$, dissolved in $\mathrm{CDCl}_{3}$, filtered through a plug of cotton wool and diluted to $5.0 \mathrm{~mL}$ with $\mathrm{CDCl}_{3}$ in a volumetric flask.

${ }^{1} \mathbf{H}$ NMR $\left(300 \mathrm{MHz}, \mathrm{CDCl}_{3}\right) \square 6.94\left(\mathrm{dd} ; \mathrm{J}_{1}=6.9 \mathrm{~Hz}, \mathrm{~J}_{2}=0.8 \mathrm{~Hz} ; 1 \mathrm{H}\right), 6.78\left(\mathrm{ddd} ; \mathrm{J}_{1}=9.6\right.$ $\left.\mathrm{Hz}, \mathrm{J}_{2}=6.3 \mathrm{~Hz}, \mathrm{~J}_{3}=1.4 \mathrm{~Hz} ; 1 \mathrm{H}\right), 6.30\left(\mathrm{dd} ; \mathrm{J}_{1}=9.6 \mathrm{~Hz}, \mathrm{~J}_{2}=0.8 \mathrm{~Hz} ; 1 \mathrm{H}\right), 5.59\left(\mathrm{ddd} ; \mathrm{J}_{1}=6.6 \mathrm{~Hz}\right.$, $\left.\mathrm{J}_{2}=6.3 \mathrm{~Hz}, \mathrm{~J}_{3}=1.1 \mathrm{~Hz} ; 1 \mathrm{H}\right), 3.88-4.03(\mathrm{~m}, 4 \mathrm{H}) ;{ }^{13} \mathbf{C} \mathbf{N M R}\left(75 \mathrm{MHz}, \mathrm{CDCl}_{3}\right) \square 158.5,136.3$, $133.7,114.8,102.4,52.0,49.6$.

\section{(R)-N-(Pyridyl-2)-2-Hydroxy-1-phenylethylamine}

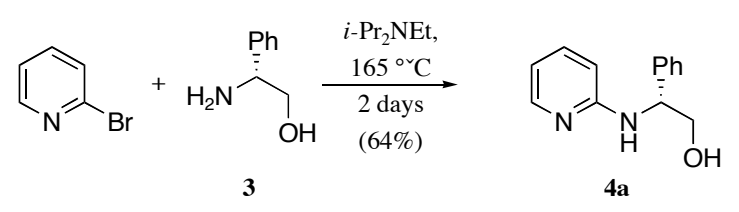

A 25-mL medium-pressure tube charged with 2-bromopyridine (158 $\mathrm{mg}, 1.00 \mathrm{mmol}),(\mathrm{R})$ phenylglycinol (137 mg, $1.00 \mathrm{mmol}), \mathrm{N}, \mathrm{N}$-diisopropylethylamine $(150 \mathrm{mg}, 1.16 \mathrm{mmol})$ and a stir bar was flushed with nitrogen several times, stoppered and heated at $160 \pm 5{ }^{\circ} \mathrm{C}$ for 2 days. The tube was allowed to cool to room temperature, the contents was diluted with a small amount of $\mathrm{CH}_{2} \mathrm{Cl}_{2}$ and chromatographed (hexanes-EtOAc 2:1 $\rightarrow$ 1:1 $\rightarrow$ neat EtOAc) to afford $137 \mathrm{mg}$ of the product, which crystallized quickly (64\% yield).

${ }^{1} \mathbf{H}$ NMR $\left(300 \mathrm{MHz}, \mathrm{CDCl}_{3}\right) \square 8.02\left(\mathrm{dd} ; J_{1}=5.2 \mathrm{~Hz}, J_{2}=0.8 \mathrm{~Hz} ; 1 \mathrm{H}\right), 7.26-7.40(\mathrm{~m}, 6 \mathrm{H}), 6.57$ $\left(\mathrm{ddd} ; J_{1}=7.2 \mathrm{~Hz}, J_{2}=5.2 \mathrm{~Hz}, J_{3}=0.8 \mathrm{~Hz} ; 1 \mathrm{H}\right), 6.29(\mathrm{~d}, J=8.5 \mathrm{~Hz} ; 1 \mathrm{H}), 5.51(\mathrm{~d}, J=4.4 \mathrm{~Hz}$, $1 \mathrm{H}), 5.28(\mathrm{~s}, \mathrm{br}, 1 \mathrm{H}), 4.78\left(\mathrm{ddd}, J_{1}=J_{2}=4.0 \mathrm{~Hz}, J_{3} 8.0 \mathrm{~Hz}, 1 \mathrm{H}\right), 3.95(\mathrm{~m}, 1 \mathrm{H}), 3.86\left(\mathrm{dd} ; J_{1}=\right.$ $\left.11.0 \mathrm{~Hz}, J_{2}=7.4 \mathrm{~Hz} ; 1 \mathrm{H}\right) ;{ }^{13} \mathbf{C} \mathbf{N M R}\left(75 \mathrm{MHz} \mathrm{CDCl}_{3}\right) \square 158.7,147.6,140.5,138.1,129.1$, 127.9, 127.0, 113.7, 108.3, 68.0, 59.6; MS: HR-FAB calculated for $\mathrm{C}_{13} \mathrm{H}_{14} \mathrm{~N}_{2} \mathrm{O}_{2}\left(\mathrm{M}+\mathrm{H}^{+}\right) \mathrm{m} / \mathrm{z}$ : 215.1184, measured m/z: 215.1181, error $=-1.5 \mathrm{ppm} ; \mathbf{m p ~} 104-106{ }^{\circ} \mathrm{C} ;[\square]_{\mathrm{D}}=-21^{\circ}(\mathrm{c}=1.05$, $\mathrm{MeOH})$.

\section{(R)-2-Phenyl-2, 3-dihydroimidazo[1,2-a]pyridine}

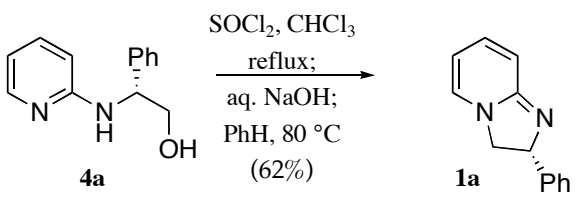

A solution of $\mathbf{4 a}(214 \mathrm{mg}, 1.00 \mathrm{mmol})$ in $2 \mathrm{~mL}$ of $\mathrm{CHCl}_{3}$ was treated with $\mathrm{SOCl}_{2}(0.150 \mathrm{~mL}$, $2.06 \mathrm{mmol}$ ) added dropwise at room temperature, then heated to reflux in an oil bath kept at 70 ${ }^{\circ} \mathrm{C}$. After 1 hour, the flask was taken out of the bath, allowed to cool and treated cautiously with 2-3 drops of $\mathrm{MeOH}$ (vigorous gas evolution!), then heated again for 5-10 minutes. The solvent was removed under reduced pressure, and the semicrystalline, gummy residue was extracted with warm water. The aqueous extract was filtered from gummy particles through a cotton plug, basified with concentrated $\mathrm{NaOH}$ and extracted with benzene (ca. $25 \mathrm{~mL}$ ). The benzene solution 
was dried briefly over $\mathrm{Na}_{2} \mathrm{SO}_{4}$ and then heated under reflux in an oil bath at $80-85{ }^{\circ} \mathrm{C}$. After 2 hours, the reaction mixture was cooled to room temperature and extracted with water 3 times. The aqueous extract was made strongly basic with concentrated $\mathrm{NaOH}$ and extracted with $\mathrm{CH}_{2} \mathrm{Cl}_{2}$. The organic extract was dried over $\mathrm{Na}_{2} \mathrm{SO}_{4}$ and evaporated to give bright-yellow, crystalline product (121 mg, 62\% yield). Recrystallization from boiling hexanes gave thin plates. ${ }^{1} \mathbf{H}$ NMR $\left(300 \mathrm{MHz}, \mathrm{CDCl}_{3}\right.$ ) $\square$ 7.24-7.35 (m, 5H), 6.90-6.93 (m, 1H), 6.82-6.88 (m, 1H), 6.42 (ddd, $\left.J_{1}=9.34 \mathrm{~Hz}, J_{2}=1.38 \mathrm{~Hz}, J_{3}=0.28 \mathrm{~Hz}, 1 \mathrm{H}\right), 5.66(\mathrm{~m}, 1 \mathrm{H}), 5.23(\mathrm{~m}, 1 \mathrm{H}), 4.36(\mathrm{~m}, 1 \mathrm{H})$, $3.84(\mathrm{~m}, 1 \mathrm{H}) ;{ }^{13} \mathbf{C} \mathbf{N M R}\left(75 \mathrm{MHz}, \mathrm{CDCl}_{3}\right) \square$ 158.4, 145.0, 137.0, 133.8, 128.8, 127.3, 126.8, 115.0, 103.2, 67.3, 57.6; MS: HR-FAB calculated for $\mathrm{C}_{13} \mathrm{H}_{13} \mathrm{~N}_{2}\left(\mathrm{M}+\mathrm{H}^{+}\right) \mathrm{m} / \mathrm{z}$ : 197.1079, measured m/z: 197.1070, error $=-4.4 \mathrm{ppm} ; \mathbf{m p ~} 101-103^{\circ} \mathrm{C} ;[\square]_{\mathrm{D}}=+412^{\circ}(\mathrm{c}=1.03, \mathrm{MeOH})$.

\section{(R)-N-(5-Bromopyridyl-2)-2-Hydroxy-1-phenylethylamine}

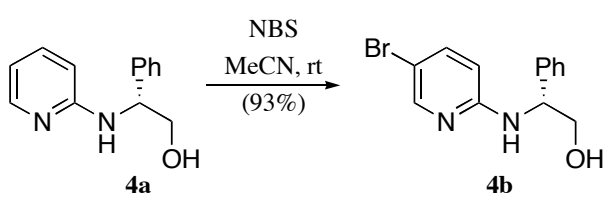

To a solution of $\mathbf{4 a}(214 \mathrm{mg}, 1.00 \mathrm{mmol})$ in $5 \mathrm{~mL}$ of $\mathrm{MeCN}$ was added, in several portions, NBS (178 mg, $1.00 \mathrm{mmol})$. The mixture was stirred at room temperature, then concentrated and chromatographed (hexanes-EtOAc 3:1 $\rightarrow 2: 1$ ) to afford $273 \mathrm{mg}$ of white solid (93\% yield).

${ }^{1} \mathbf{H}$ NMR $\left(300 \mathrm{MHz}, \mathrm{CDCl}_{3}\right) \square 8.07(\mathrm{~d}, 2.47 \mathrm{~Hz}, 1 \mathrm{H}), 7.41\left(\mathrm{dd}, J_{1}=8.79 \mathrm{~Hz}, J_{2}=2.47 \mathrm{~Hz} ; 1 \mathrm{H}\right)$, 7.26-7.37 (m, 5H), $6.24(\mathrm{~d}, J=9.07 \mathrm{~Hz}, 1 \mathrm{H}), 5.36(\mathrm{~d}, J=5.49 \mathrm{~Hz}, 1 \mathrm{H}), 4.79(\mathrm{~m}, 2 \mathrm{H}), 3.92(\mathrm{~m}$, 2H), 3.02 (s, br, 1H) ; ${ }^{13} \mathbf{C}$ NMR (75 MHz, $\left.\mathrm{CDCl}_{3}\right) \square 157.1,148.5,140.3,139.9,129.2$, 128.2, 127.0, 109.7, 108.0, 67.8, 59.2; MS: HR-FAB calculated for $\mathrm{C}_{13} \mathrm{H}_{13} \mathrm{BrN}_{2} \mathrm{O}\left(\mathrm{M}+\mathrm{H}^{+}\right) \mathrm{m} / \mathrm{z}$ : 293.0281, measured m/z: 293.0275, error $=-4.9 \mathrm{ppm} ; \mathbf{m p ~} 100-102{ }^{\circ} \mathrm{C}$; $[\square]_{\mathrm{D}}=-73^{\circ}(\mathrm{c}=1.07$, $\mathrm{MeOH})$.

\section{(R)-5-Bromo-2-phenyl-2, 3-dihydroimidazo[1,2-a]pyridine}

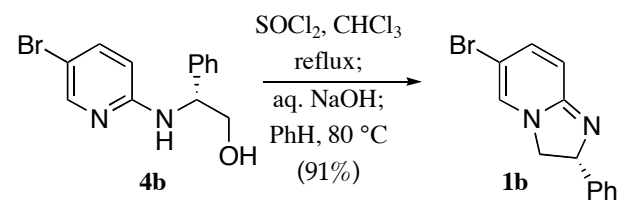

A procedure analogous to the cyclization of $4 \mathbf{a}$ was carried out on $0.5 \mathrm{mmol}$ scale $(147 \mathrm{mg}$ of $4 \mathbf{b}, 0.075 \mathrm{~mL} \mathrm{SOCl}_{2}, 1 \mathrm{~mL} \mathrm{CHCl}_{3}$ ) and produced $126 \mathrm{mg}$ (91\% yield) of yellow oil which crystallized quickly.

${ }^{1} \mathbf{H}$ NMR $\left(300 \mathrm{MHz}, \mathrm{CDCl}_{3}\right) \square 7.26-7.37(\mathrm{~m}, 5 \mathrm{H}), 7.07(\mathrm{~d}, J=1.37 \mathrm{~Hz}, 1 \mathrm{H}), 6.86\left(\mathrm{dd}, J_{1}=9.89\right.$ $\left.\mathrm{Hz}, J_{2}=2.20 \mathrm{~Hz}, 1 \mathrm{H}\right), 6.37\left(\mathrm{dd}, J_{1}=11.70 \mathrm{~Hz}, J_{2}=0.55 \mathrm{~Hz}, 1 \mathrm{H}\right), 5.25(\mathrm{dd}, J=11.54 \mathrm{~Hz}, 1 \mathrm{H})$, $4.35(\mathrm{t}, J=10.98,1 \mathrm{H}), 3.86\left(\mathrm{dd}, J_{1}=9.07 \mathrm{~Hz}, J_{2}=10.80 \mathrm{~Hz}, 1 \mathrm{H}\right) ;{ }^{13} \mathbf{C ~ N M R}\left(75 \mathrm{MHz}, \mathrm{CDCl}_{3}\right) \square$ 156.5, 144.3, 140.0, 133.7, 129.0, 127.6, 126.8, 116.3, 94.3, 67.7, 57.8; MS: HR-FAB calculated for $\mathrm{C}_{13} \mathrm{H}_{12} \mathrm{BrN}_{2}\left(\mathrm{M}+\mathrm{H}^{+}\right) \mathrm{m} / \mathrm{z}: 275.0184$, measured m/z: 275.0172, error = $-4.3 \mathrm{ppm}$; mp $86-87.5^{\circ} \mathrm{C} ;[\mathrm{[}]_{\mathrm{D}}=+353^{\circ}(\mathrm{c}=1.03, \mathrm{MeOH})$. 
(R)-N-(5-Nitropyridyl-2)-2-Hydroxy-1-phenylethylamine

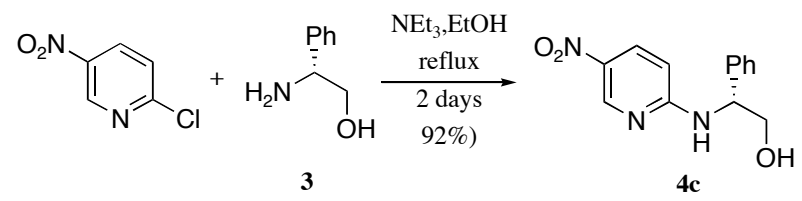

A solution of 2-chloro-5-nitropyridine (316 mg, $2.00 \mathrm{mmol}),(\mathrm{R})$-phenylglycinol (274 $\mathrm{mg}, 2.00 \mathrm{mmol})$ and $\mathrm{NEt}_{3}(0.280 \mathrm{~mL}, 2.00 \mathrm{mmol})$ in $3.5 \mathrm{~mL}$ of absolute EtOH was refluxed under nitrogen for 2 days. EtOH was removed on a rotary evaporator and the residue was chromatographed (hexanes-EtOAc 3:1 $\rightarrow 1: 1$ ) to afford $480 \mathrm{mg}$ of the product as orange, viscous oil, which crystallized after long standing (92\% yield).

${ }^{1} \mathbf{H}$ NMR $\left(300 \mathrm{MHz}, \mathrm{CDCl}_{3}\right) \square 8.96(\mathrm{~d}, J=2.7 \mathrm{~Hz}, 1 \mathrm{H}), 8.12\left(\mathrm{dd}, J_{1}=9.1 \mathrm{~Hz}, J_{2}=2.7 \mathrm{~Hz}, 1 \mathrm{H}\right)$, 7.26-7.41 (m, 5H), $6.31(\mathrm{~d}, J=9.1 \mathrm{~Hz}, 2 \mathrm{H}), 5.00(\mathrm{~s}, \mathrm{br}, 1 \mathrm{H}), 4.04\left(\mathrm{dd}, J_{1}=11.3 \mathrm{~Hz}, J_{2}=3.8 \mathrm{~Hz}\right.$, $1 \mathrm{H}), 3.95\left(\mathrm{dd}, J_{1}=11.3 \mathrm{~Hz}, J_{2}=5.8 \mathrm{~Hz}, 1 \mathrm{H}\right), 2.38(\mathrm{~s}, \mathrm{br}, 1 \mathrm{H}) ;{ }^{13} \mathbf{C} \mathbf{N M R}\left(75 \mathrm{MHz}, \mathrm{CDCl}_{3}\right) \square$ $160.2,145.9(\mathrm{q}, J=4.5 \mathrm{~Hz}), 139.5,134.9(\mathrm{q}, J=1.5 \mathrm{~Hz}), 129.3,128.3,126.9,124.6(\mathrm{q}, J=270$ $\mathrm{Hz}), 116.4$ (q, $J=33.2 \mathrm{~Hz}), 107.4,67.5,58.8$; MS: HR-FAB calculated for $\mathrm{C}_{13} \mathrm{H}_{13} \mathrm{BrN}_{2} \mathrm{O}(\mathrm{M}+$ $\left.\mathrm{H}^{+}\right) \mathrm{m} / \mathrm{z}: 260.1035$, measured m/z: 260.1057, error $=-4.4 \mathrm{ppm} ; \mathbf{m p 1 0 9 - 1 1 0}{ }^{\circ} \mathrm{C} ;[\mathrm{C}]_{\mathrm{D}}=-165^{\circ}(\mathrm{c}$ $=0.96, \mathrm{MeOH})$.

\section{(R)-5-Nitro-2-phenyl-2, 3-dihydroimidazo[1,2-a]pyridine}

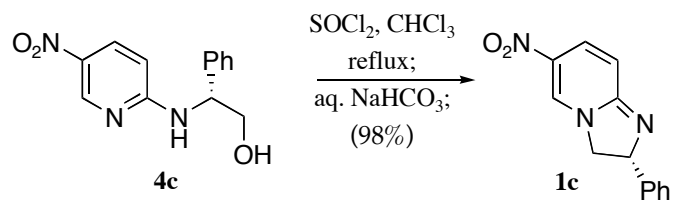

A solution of $4 \mathbf{c}(347 \mathrm{mg}, 1.34 \mathrm{mmol})$ in $7 \mathrm{~mL}$ of $\mathrm{CHCl}_{3}$ was treated with $\mathrm{SOCl}_{2}(0.250 \mathrm{~mL}$, $3.43 \mathrm{mmol}$ ) added dropwise at room temperature, then heated to reflux in an oil bath kept at 65 ${ }^{\circ} \mathrm{C}$. After 1.5 hours, the flask was taken out of the bath, allowed to cool somewhat and treated cautiously with 2-3 drops of $\mathrm{MeOH}$ (vigorous gas evolution!), then heated again for 5 minutes. The mixture was rotary evaporated and the evaporation residue was extracted with water. The aqueous extract was decanted from the gummy residue, brought to $\mathrm{pH} 7-8$ with aqueous $\mathrm{NaHCO}_{3}$ and extracted with $\mathrm{CH}_{2} \mathrm{Cl}_{2} 3$ times. More aqueous $\mathrm{NaHCO}_{3} / \mathrm{NaOH}$ was added to the aqueous phase to $\mathrm{pH} 12$ and extraction was continued until organic extracts were pale-yellow (4 times). The organic phase was dried over $\mathrm{NaOH}$ pellets and then rotary evaporated. The crude mixture was chromatographed $\left(20 \% i-\mathrm{PrOH}+2 \% \mathrm{NEt}_{3}\right.$ in hexanes) to give $318 \mathrm{mg}$ of lightorange, non-crystalline mass $(98 \%)$.

${ }^{1} \mathbf{H}$ NMR $\left(300 \mathrm{MHz}, \mathrm{CDCl}_{3}\right) \square 8.38(\mathrm{~d}, J=2.20 \mathrm{~Hz}, 1 \mathrm{H}), 7.59\left(\mathrm{dd}, J_{1}=10.44 \mathrm{~Hz}, J_{2}=7.97 \mathrm{~Hz}\right.$, $1 \mathrm{H}), 7.26-7.38(\mathrm{~m}, 5 \mathrm{H}), 6.38(\mathrm{~d}, J=10.44 \mathrm{~Hz}, 1 \mathrm{H}), 5.37\left(\mathrm{dd}, J_{1}=10.71 \mathrm{~Hz}, J_{2}=8.24 \mathrm{~Hz}, 1 \mathrm{H}\right)$, $4.46(\mathrm{t}, J=11.54,1 \mathrm{H}), 3.94\left(\mathrm{dd}, J_{1}=11.82 \mathrm{~Hz}, J_{2}=8.24 \mathrm{~Hz}, 1 \mathrm{H}\right) ;{ }^{13} \mathbf{C} \mathbf{N M R}\left(75 \mathrm{MHz}, \mathrm{CDCl}_{3}\right) \square$ 155.2, 142.5, 137.8, 130.9, 129.5, 129.1, 128.1, 126.7, 113.4, 68.8 57.1; MS: HR-FAB calculated for $\mathrm{C}_{13} \mathrm{H}_{12} \mathrm{~N}_{3} \mathrm{O}_{2}\left(\mathrm{M}+\mathrm{H}^{+}\right) \mathrm{m} / \mathrm{z}: 242.0930$, measured $\mathrm{m} / \mathrm{z}$ : 242.0930, error = $0.2 \mathrm{ppm}$; $[\square]_{\mathrm{D}}=+173^{\circ}(\mathrm{c}=0.94, \mathrm{MeOH})$. 
(R)-N-(5-Trifluoromethylpyridyl-2)-2-Hydroxy-1-phenylethylamine

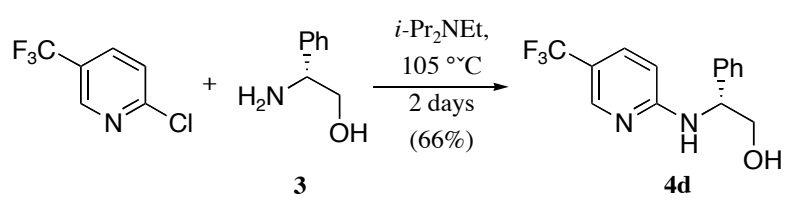

A $25 \mathrm{~mL}$ medium-pressure tube charged with 2-chloro-5-trifluoromethylpyridine (1.502 g, $8.270 \mathrm{mmol}),(\mathrm{R})$-phenylglycinol (1.102 g, $8.030 \mathrm{mmol}), \mathrm{N}, \mathrm{N}$-diisopropylethylamine $(1.200 \mathrm{~g}$, $9.300 \mathrm{mmol}$ ) and a stir bar was flushed with nitrogen several times, stoppered and heated at 105 $\pm 5^{\circ} \mathrm{C}$ for 2 days. The tube was allowed to cool to room temperature, the contents was diluted with a small amount of $\mathrm{CH}_{2} \mathrm{Cl}_{2}$ and chromatographed (isopropanol-hexanes $5 \% \rightarrow 10 \%$ ) to afford $1.503 \mathrm{~g}$ of the product, which crystallized quickly (66\% yield).

${ }^{1} \mathbf{H}$ NMR $\left(300 \mathrm{MHz}, \mathrm{CDCl}_{3}\right) \square 8.28(\mathrm{~s}, 1 \mathrm{H}), 7.52\left(\mathrm{dd}, J_{1}=8.79 \mathrm{~Hz}, J_{2}=2.20 \mathrm{~Hz}, 1 \mathrm{H}\right), 7.26-7.37$ $(\mathrm{m}, 5 \mathrm{H}), 6.33(\mathrm{~d}, J=8.79 \mathrm{~Hz}, 1 \mathrm{H}), 5.80(\mathrm{~d}, J=5.50 \mathrm{~Hz}, 1 \mathrm{H}), 4.89\left(\mathrm{dd}, J_{1}=9.89 \mathrm{~Hz}, J_{2}=6.05\right.$ $\mathrm{Hz}, 1 \mathrm{H}), 3.99\left(\mathrm{dd}, J_{1}=11.26 \mathrm{~Hz}, J_{2}=3.84 \mathrm{~Hz}, 1 \mathrm{H}\right), 3.91\left(\mathrm{dd}, J_{1}=10.99 \mathrm{~Hz}, J_{2}=6.60 \mathrm{~Hz}, 1 \mathrm{H}\right)$, 3.28 (s, br, 1H); ${ }^{13} \mathbf{C}$ NMR (75 MHz, $\left.\mathrm{CDCl}_{3}\right) \square$ 161.0, 146.9, 138.7, 136.5, 133.4, 129.4, 128.5, 126.8, 106.7, 66.9, 58.3; MS: HR-FAB calculated for $\mathrm{C}_{13} \mathrm{H}_{13} \mathrm{BrN}_{2} \mathrm{O}\left(\mathrm{M}+\mathrm{H}^{+}\right) \mathrm{m} / \mathrm{z}$ : 283.1058, measured m/z: 283.1069 , error $=3.8 \mathrm{ppm} ; \mathbf{m p} 105-105.5^{\circ} \mathrm{C} ;[\square]_{\mathrm{D}}=-57^{\circ}(\mathrm{c}=1.09, \mathrm{MeOH})$.

\section{(R)-5-Trifluoromethyl-2-phenyl-2, 3-dihydroimidazo[1,2-a]pyridine}
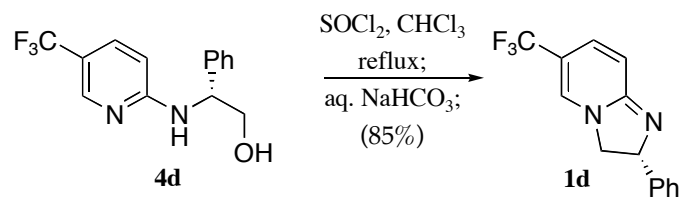

A procedure analogous to the cyclization of $4 \mathbf{c}$ was carried out on $5.24 \mathrm{mmol}$ scale $(1.499 \mathrm{~g}$ of $\mathbf{4 d}, 1.05 \mathrm{~mL} \mathrm{SOCl}_{2}, 30 \mathrm{~mL} \mathrm{CHCl}_{3}$ ) and produced after chromatography (isopropanol-hexanes $5 \% \rightarrow 10 \%) 1.176 \mathrm{~g}(85 \%$ yield) of yellow oil which crystallized quickly.

Recrystallization from hexanes produced yellow needles (78\% recovery). Note: for the sake of consistency, all the data for kinetic resolution experiments shown in the table were obtained using the recrystallized material. However, the difference between it and the unrecrystallized product becomes appreciable only at selectivity levels of 25:1 and above.

${ }^{1}$ H NMR $\left(300 \mathrm{MHz}, \mathrm{CDCl}_{3}\right) \square 7.26-7.38(\mathrm{~m}, 6 \mathrm{H}) 6.93\left(\mathrm{dd}, J_{1}=10.01 \mathrm{~Hz}, J_{2}=2.20 \mathrm{~Hz}, 1 \mathrm{H}\right) 6.46$ (d, $J=9.77,1 \mathrm{H}) 5.29\left(\mathrm{dd}, J_{1}=11.48 \mathrm{~Hz}, J_{2}=8.79 \mathrm{~Hz}, 1 \mathrm{H}\right), 4.36(\mathrm{t}, J=11.23 \mathrm{~Hz}, 1 \mathrm{H}), 3.86(\mathrm{t}, J$ $=9.74 \mathrm{~Hz}, 1 \mathrm{H}) ;{ }^{13} \mathbf{C}$ NMR $\left(75 \mathrm{MHz}, \mathrm{CDCl}_{3}\right) \square 156.7,143.8,133.8(\mathrm{q}, J=5.54 \mathrm{~Hz}), 132.5(\mathrm{q}, J=$ $2.51 \mathrm{~Hz}), 129.0,127.8,126.8,124.0(\mathrm{q}, J=268 \mathrm{~Hz}), 115.6,106.9(\mathrm{q}, J=34.8 \mathrm{~Hz}), 67.9,57.2$; MS: HR-FAB calculated for $\mathrm{C}_{14} \mathrm{H}_{12} \mathrm{~F}_{3} \mathrm{LiN}_{2}\left(\mathrm{M}+\mathrm{Li}^{+}\right) \mathrm{m} / \mathrm{z}: 271.1034$, measured m/z: 271.1029, error $=-1.9 \mathrm{ppm} ; \mathbf{m p ~} 128-129^{\circ} \mathrm{C}($ from hexanes $) ;[\square]_{\mathrm{D}}=+277^{\circ}(\mathrm{c}=0.99, \mathrm{MeOH})$. 


\section{C) Kinetic resolution experiments.}

All kinetic resolution experiments were carried out according to Procedures A, B, C, and D described below. Selectivities and conversions were determined as described in Procedure E.

\section{Procedure A. Variation of substituent X in DHIP catalysts.}

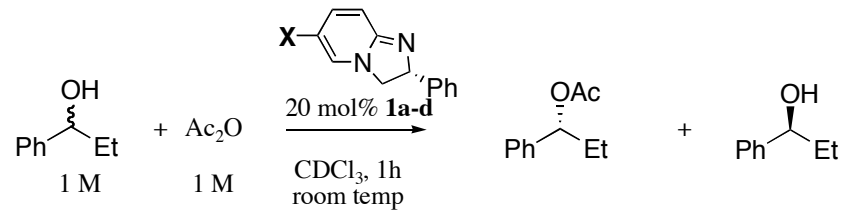

To a solution of $0.25 \mathrm{mmol}$ of phenylethylcarbinol (34 $\square \mathrm{L}, 34 \mathrm{mg}$ ) and $0.050 \mathrm{mmol}$ of the catalyst (1a-d) in $0.250 \mathrm{~mL} \mathrm{CDCl}_{3}$ was added $0.25 \mathrm{mmol}$ of acetic anhydride (24 $\square \mathrm{L}, 26 \mathrm{mg}$ ). The mixture was swirled, left at room temperature for 1 hour, quenched by rapid addition of 0.25 $\mathrm{mL}$ of methanol, and left for one more hour. The reaction mixture was diluted with $\mathrm{CH}_{2} \mathrm{Cl}_{2}$, washed twice with $1 \mathrm{M} \mathrm{HCl}$, then twice with saturated aqueous $\mathrm{NaHCO}_{3}$, and dried over $\mathrm{Na}_{2} \mathrm{SO}_{4}$. The solution was concentrated on a rotary evaporator at room temperature and chromatographed $\left(5-20 \% \mathrm{Et}_{2} \mathrm{O}\right.$ in hexanes) to separate the ester from the unreacted alcohol.

Table 1.

\begin{tabular}{|c|c|c|c|c|c|c|c|c|c|}
\hline Entry & $\mathbf{X}$ & $\mathbf{t}(\mathbf{h})$ & $\#$ & $\begin{array}{c}\mathbf{e e}_{\mathrm{E}} \\
\%\end{array}$ & $\begin{array}{c}\mathbf{e e}_{\mathrm{A}} \\
\%\end{array}$ & $\begin{array}{c}\mathbf{C}_{\text {HPLC }} \\
\%\end{array}$ & $\mathbf{S}$ & $\begin{array}{c}\mathrm{C}_{\mathrm{AVG}} \\
\%\end{array}$ & $\mathbf{S}_{\mathrm{AVG}}$ \\
\hline \multirow{2}{*}{1} & \multirow{2}{*}{$\mathrm{H}$} & \multirow{2}{*}{1.0} & 1 & 49.0 & 12.8 & 20.7 & 3.3 & \multirow{2}{*}{21} & \multirow{2}{*}{3.3} \\
\hline & & & 2 & 49.5 & 13.4 & 21.1 & 3.36 & & \\
\hline \multirow{2}{*}{2} & \multirow{2}{*}{$\mathrm{Br}$} & \multirow{2}{*}{1.0} & 1 & 74.4 & 25.2 & 25.3 & 8.7 & \multirow{2}{*}{25} & \multirow{2}{*}{8.6} \\
\hline & & & 2 & 74.0 & 24.4 & 24.8 & 8.5 & & \\
\hline \multirow{2}{*}{3} & \multirow{2}{*}{$\mathrm{NO}_{2}$} & \multirow{2}{*}{1.0} & 1 & 81.7 & 13.4 & 14.1 & 11.3 & \multirow{2}{*}{14} & \multirow{2}{*}{11} \\
\hline & & & 2 & 81.5 & 12.0 & 12.9 & 11.1 & & \\
\hline \multirow{2}{*}{4} & \multirow{2}{*}{$\mathrm{CF}_{3}$} & \multirow{2}{*}{1.0} & 1 & 79.5 & 51.9 & 39.5 & 14.6 & \multirow{2}{*}{38} & \multirow{2}{*}{14} \\
\hline & & & 2 & 79.4 & 46.3 & 36.8 & 13.7 & & \\
\hline
\end{tabular}

Procedure B. Variation of the substrate and the acylating agent.

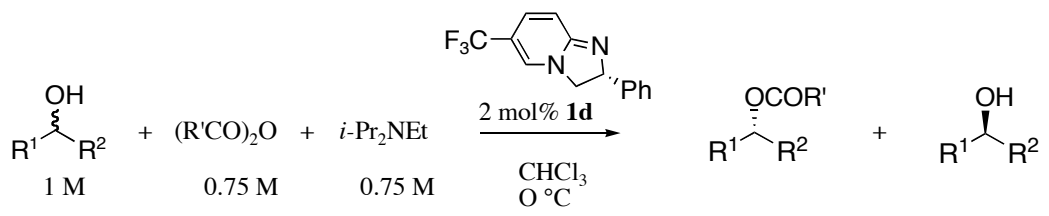

1) The stock solution of the catalyst was prepared by dissolving $0.100 \mathrm{mmol}$ of $\mathbf{1 d}(26.4$ $\mathrm{mg}$ ) and $3.75 \mathrm{mmol}$ of $\mathrm{N}, \mathrm{N}$-diisopropylethylamine $\left(654 \mathrm{LL}, 485 \mathrm{mg}\right.$ ) in $\mathrm{CHCl}_{3}$ in a $5 \mathrm{~mL}$ volumetric flask and bringing the volume to the mark. 
2) A one-dram vial was charged with $0.5 \mathrm{mmol}$ of the racemic secondary alcohol and 0.500 $\mathrm{mL}$ of the stock solution of $\mathbf{1 d}$, and cooled in an ice bath. After 15 minutes, $0.375 \mathrm{mmol}$ of the anhydride was added. The mixture was swirled and left in the ice bath for a specified period of time, at the end of which it was quenched by rapid addition of $0.5 \mathrm{~mL}$ of methanol, allowed to warm slowly and left for one more hour at room temperature. The workup and chromatography were carried out as described in Procedure A.

Table 2.

\begin{tabular}{|c|c|c|c|c|c|c|c|c|c|c|c|}
\hline Entry & $\mathbf{R}^{1}$ & $\mathbf{R}^{2}$ & $\mathbf{R}^{\prime}$ & $\mathbf{t}(\mathbf{h})$ & \# & $\begin{array}{c}\mathbf{e e}_{\mathrm{E}} \\
\%\end{array}$ & $\begin{array}{c}\mathbf{e e}_{\mathrm{A}} \\
\%\end{array}$ & $\begin{array}{c}\mathbf{C}_{\text {HPLC }} \\
\%\end{array}$ & $\mathbf{S}$ & $\begin{array}{c}\mathbf{C}_{\mathrm{AVG}} \\
\%\end{array}$ & $\mathbf{S}_{\mathrm{AVG}}$ \\
\hline \multirow{2}{*}{1} & \multirow{2}{*}{ Phenyl } & \multirow{2}{*}{$\mathrm{Me}$} & \multirow{2}{*}{$\mathrm{Me}$} & \multirow{2}{*}{8} & 1 & 72.9 & 20.0 & 21.5 & 7.75 & \multirow{2}{*}{21} & \multirow{2}{*}{7.7} \\
\hline & & & & & 2 & 73.0 & 18.9 & 20.6 & 7.70 & & \\
\hline \multirow{2}{*}{2} & \multirow[t]{2}{*}{ “ } & \multirow{2}{*}{ Et } & \multirow{2}{*}{$\mathrm{Me}$} & \multirow{2}{*}{8} & 1 & 80.7 & 60.6 & 42.9 & 17.2 & \multirow{2}{*}{43} & \multirow{2}{*}{17} \\
\hline & & & & & 2 & 81.0 & 59.6 & 42.4 & 17.4 & & \\
\hline \multirow{2}{*}{3} & \multirow[t]{2}{*}{ “ } & \multirow{2}{*}{$i-\operatorname{Pr}$} & \multirow{2}{*}{$\mathrm{Me}$} & \multirow{2}{*}{30} & 1 & 82.4 & 79.2 & 49.0 & 24.8 & \multirow{2}{*}{47} & \multirow{2}{*}{24} \\
\hline & & & & & 2 & 82.9 & 70.3 & 45.9 & 22.4 & & \\
\hline \multirow{2}{*}{4} & \multirow[t]{2}{*}{ “ } & \multirow{2}{*}{$\mathrm{Me}$} & \multirow{2}{*}{ Et } & \multirow{2}{*}{8} & 1 & 89.3 & 42.7 & 32.3 & 26.8 & \multirow{2}{*}{32} & \multirow{2}{*}{26} \\
\hline & & & & & 2 & 89.2 & 40.3 & 31.1 & 26.0 & & \\
\hline \multirow{2}{*}{5} & “ & & Ft & 8 & 1 & 90.4 & 60.8 & 40.2 & 36.8 & 30 & 36 \\
\hline & 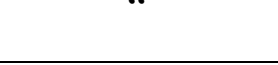 & Et & $\mathrm{Et}$ & 8 & 2 & 90.6 & 57.2 & 38.7 & 36.0 & 39 & 50 \\
\hline & “ & & & & 1 & 80.9 & 97.6 & 54.7 & 41.1 & & \\
\hline 6 & . & $l-\mathrm{Pr}$ & Et & 30 & 2 & 78.7 & 98.9 & 55.7 & 41.8 & 55 & 41 \\
\hline & “ & & & & 1 & 93.5 & 88.2 & 48.6 & 87.1 & & \\
\hline 1 & 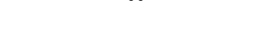 & $t$-Bu & $\mathrm{Et}$ & 52 & 2 & 93.8 & 83.6 & 47.1 & 82.5 & 48 & 85 \\
\hline 8 & 1 Nonhtbul & Mo & Et & 8 & 1 & 90.1 & 89.1 & 49.7 & 57.8 & $51+2>>$ & 56 \\
\hline 0 & 1-1Napnitiyi & IVIE & $\mathrm{Ll}$ & 8 & 2 & 87.8 & 94.0 & 51.7 & 54.4 & J1 & 50 \\
\hline 0 & $m-\mathrm{MeCH}$ & Me & Ft & 8 & 1 & 88.1 & 51.7 & 37.0 & 26.3 & 36 & 27 \\
\hline 9 & $m$-IMIeC ${ }_{6} \mathbf{H}_{4}$ & Mie & El & 8 & 2 & 88.7 & 48.8 & 35.5 & 27.1 & 30 & 21 \\
\hline 10 & $m-\mathrm{MeOC} \mathrm{H}$ & Me & Ft & 8 & 1 & 89.8 & 57.8 & 39.2 & 33.2 & 40 & 34 \\
\hline 10 & $m$-NMeU ${ }_{6} \mathbf{H}_{4}$ & Mie & $\mathrm{El}$ & 8 & 2 & 89.4 & 63.2 & 41.4 & 34.2 & 40 & 34 \\
\hline 11 & $m_{-} \mathrm{BrCH}$ & Me & Ft & 8 & 1 & 88.1 & 69.1 & 44.0 & 32.6 & 44 & 32 \\
\hline 11 & $m-\mathrm{DIC}_{6} \mathrm{\Pi}_{4}$ & IVIE & $\mathrm{Ll}$ & 0 & 2 & 87.8 & 69.7 & 44.2 & 32.0 & 44 & 52 \\
\hline 12 & $\mathrm{MeC} \mathrm{H}$ & Mo & Et & 8 & 1 & 86.4 & 66.3 & 43.4 & 27.3 & 14 & 26 \\
\hline 12 & $O$-NIEC ${ }_{6} \Pi_{4}$ & IVIE & $\mathrm{Ll}$ & 0 & 2 & 85.5 & 66.0 & 43.6 & 25.3 & 44 & 20 \\
\hline 13 & $246 \mathrm{Me} C \mathrm{H}$ & Me & Ft & 30 & 1 & 76.2 & 85.1 & 52.8 & 19.8 & 53 & 20 \\
\hline $1 J$ & $2,+, 0$ & IVIC & $\mathrm{Lt}$ & 30 & 2 & 76.0 & 88.1 & 53.7 & 21.0 & 53 & 20 \\
\hline 14 & Cyclohexyl & $\mathrm{Me}$ & Et & 50 & $\mathrm{n} / \mathrm{a}$ & $\mathrm{nd}^{\mathrm{a}}$ & $\mathrm{nd}^{\mathrm{a}}$ & $\mathrm{nd}^{\mathrm{a}}$ & $\mathrm{nd}^{\mathrm{a}}$ & $<4^{\mathrm{b}}$ & $\mathrm{nd}^{\mathrm{a}}$ \\
\hline 15 & 1-Indanol & & Et & 50 & $\mathrm{n} / \mathrm{a}$ & $\square 0$ & $\square 0$ & $\mathrm{nd}^{\mathrm{a}}$ & $\square 1$ & $16^{\mathrm{b}}$ & $\square 1$ \\
\hline
\end{tabular}

$\left.{ }^{a}\right)$ Not determined ${ }^{b}$ ) Determined by ${ }^{1} \mathrm{H}$ NMR 
Procedure C. Variation of the solvent.

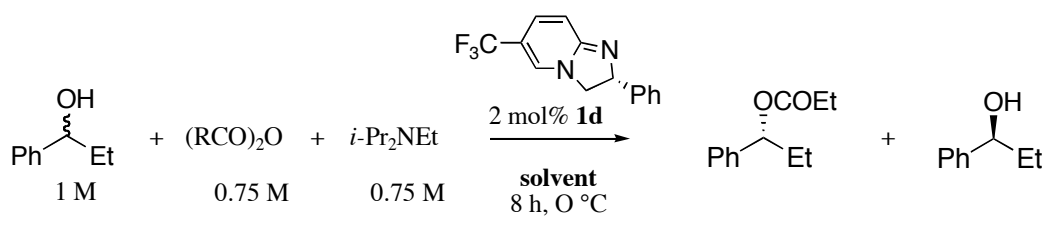

1) The stock solution of the catalyst was prepared by dissolving $0.040 \mathrm{mmol}$ of $\mathbf{1 d}$ (10.6 $\mathrm{mg}$ ) and $1.5 \mathrm{mmol}$ of $\mathrm{N}, \mathrm{N}$-diisopropylethylamine $(262 \square \mathrm{L}, 194 \mathrm{mg})$ in the reaction solvent in a 2 $\mathrm{mL}$ volumetric test tube and bringing the volume to the mark.

2) A one-dram vial was charged with $0.5 \mathrm{mmol}$ of ( \pm )-phenylethylcarbinol and $0.500 \mathrm{~mL}$ of the stock solution of $\mathbf{1 d}$, and cooled in an ice bath. After 15 minutes, $0.375 \mathrm{mmol}$ of propionic anhydride was added. The mixture was swirled and left in the ice bath for 8 hours, at the end of which it was quenched by rapid addition of $0.5 \mathrm{~mL}$ of methanol, allowed to warm slowly and left for one more hour at room temperature. The workup and chromatography were carried out as described in Procedure A.

Table 3.

\begin{tabular}{|c|c|c|c|c|c|c|c|c|}
\hline Entry & Solvent & \# & $\begin{array}{c}\mathbf{e e}_{\mathrm{E}} \\
\%\end{array}$ & $\begin{array}{c}\mathbf{e e}_{\mathrm{A}} \\
\%\end{array}$ & $\begin{array}{c}\mathbf{C}_{\text {HPLC }} \\
\% \%\end{array}$ & $\mathbf{s}$ & $\begin{array}{c}\mathbf{C}_{\mathrm{AVG}} \\
\%\end{array}$ & $\mathbf{S}_{\mathrm{AVG}}$ \\
\hline \multirow{2}{*}{1} & \multirow{2}{*}{ Diethyl ether } & 1 & 93.5 & 35.8 & 27.7 & 42.0 & \multirow{2}{*}{27} & \multirow{2}{*}{40} \\
\hline & & 2 & 92.9 & 34.6 & 27.1 & 38.3 & & \\
\hline \multirow{2}{*}{2} & \multirow{2}{*}{ Toluene } & 1 & 92.8 & 36.7 & 28.3 & 38.3 & \multirow{2}{*}{30} & \multirow{2}{*}{36} \\
\hline & & 2 & 91.6 & 41.7 & 31.3 & 34.5 & & \\
\hline \multirow{2}{*}{3} & \multirow{2}{*}{ Dichloromethane } & 1 & 88.4 & 40.3 & 31.3 & 24.2 & \multirow{2}{*}{30} & \multirow{2}{*}{24} \\
\hline & & 2 & 89.2 & 34.7 & 28.0 & 24.5 & & \\
\hline \multirow[t]{2}{*}{4} & \multirow{2}{*}{ t-Amyl alcohol } & 1 & 90.4 & 21.4 & 19.2 & 24.3 & \multirow[b]{2}{*}{18} & \multirow{2}{*}{23} \\
\hline & & 2 & 89.9 & 18.7 & 17.2 & 22.5 & & \\
\hline \multirow[b]{2}{*}{$J$} & \multirow{2}{*}{ Acetonitrile } & 1 & 81.5 & 19.5 & 19.3 & 11.8 & \multirow{2}{*}{20} & \multirow{2}{*}{11} \\
\hline & & 2 & 79.3 & 19.6 & 19.8 & 10.5 & & \\
\hline
\end{tabular}

Procedure D. Preparative scale resolution of ( \pm )-1-(1-naphthyl)-1-ethanol.

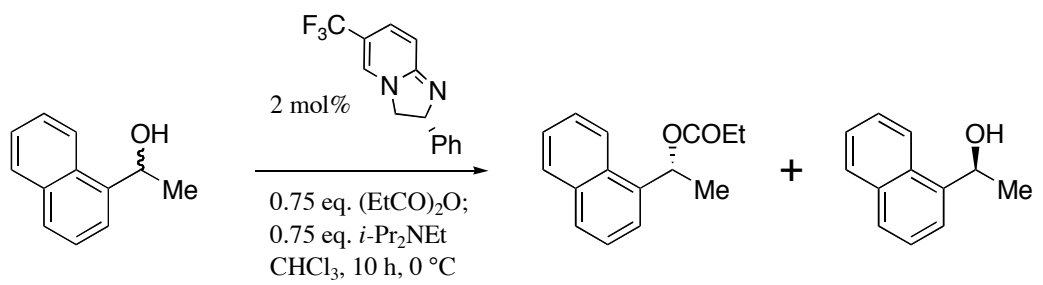

The same proportions were used as in Procedure B described above. A solution of the substrate $(2.416 \mathrm{~g}, 14.0 \mathrm{mmol})$, DIPEA $(1.93 \mathrm{~mL}, 10.5 \mathrm{mmol})$ and $\mathrm{CF}_{3}-\mathrm{PIP} 2 \mathrm{2d}(74 \mathrm{mg}, 0.28$ $\mathrm{mmol})$ in $14 \mathrm{~mL}$ of chloroform was stirred magnetically in an ice bath for 15 minutes, then 
treated with propionic anhydride $(1.35 \mathrm{~mL}, 10.5 \mathrm{mmol})$. The mixture was stirred at $0{ }^{\circ} \mathrm{C}$ for 10 hours, at which time it was quenched with methanol $(10 \mathrm{~mL})$, allowed to warm slowly and left for one more hour at room temperature. The reaction mixture was diluted with $\mathrm{CH}_{2} \mathrm{Cl}_{2}$, washed twice with $1 \mathrm{M} \mathrm{HCl}$, then twice with saturated aqueous $\mathrm{NaHCO}_{3}$, and dried over $\mathrm{Na}_{2} \mathrm{SO}_{4}$. The solution was concentrated on a rotary evaporator at room temperature and chromatographed (5 $20 \% \mathrm{Et}_{2} \mathrm{O}$ in hexanes). The ester was eluted first (1.672 g, $7.32 \mathrm{mmol}, 52 \%$ yield), followed by the unreacted alcohol (1.091 g, $6.33 \mathrm{mmol}, 45 \%$ yield $)$. The enantiomeric excess of the ester was determined by HPLC (vide infra) to be $82.5 \%$, and that of the alcohol, $98.8 \%$. Based on these ee values, the conversion was calculated to be $54.5 \%$ ( $c f .53 .6 \%$ conversion based on the isolated materials), and the selectivity factor, 52.3. The aqueous phase obtained during the workup was basified with $0.5 \mathrm{M} \mathrm{NaOH}$ and extracted with $\mathrm{CH}_{2} \mathrm{Cl}_{2}$ several times until the aqueous phase was pale-yellow. The extract was dried over $\mathrm{Na}_{2} \mathrm{SO}_{4}$, evaporated, and chromatographed to give $50 \mathrm{mg}$ of pure $\mathrm{CF}_{3}$-PIP (68\% recovery).

\section{Procedure E. Determination of Enantiomeric Excesses, Conversions and Selectivities.}

All the alcohols recovered from kinetic resolutions were found to be enriched in the Senantiomer according to the negative sign of optical rotation. Accordingly, the esters were concluded to be enriched in the R-enantiomer.

The enantiomeric excesses of the alcohols were determined by HPLC using a CHIRALCEL OD-H analytical column $(4.6 \times 250 \mathrm{~mm}$, Chiral Technologies, Inc.) and 3\% isopropanol in hexanes as mobile phase, except for 1-(1-naphthyl)-1-ethanol, which required $10 \%$ isopropanol in hexanes.

The enantiomeric excesses of the esters were determined, in most cases, by hydrolysis to the parent alcohols ( $2 \mathrm{~mL}$ of $2 \mathrm{M} \mathrm{KOH}$ in methanol, at room temperature until complete by TLC), which were analyzed as described above. 1-(1-naphthyl)-1-ethyl propionate was analyzed directly on CHIRALCEL OD-H analytical column using 3\% isopropanol in hexanes.

The enantioselectivity was calculated according to the equation:

$$
\mathrm{S}=\ln \left(\left(1-\mathrm{C}_{\mathrm{HPLC}}\right)\left(1-\mathrm{ee}_{\mathrm{A}}\right)\right) / \ln \left(\left(1-\mathrm{C}_{\mathrm{HPLC}}\right)\left(1+\mathrm{ee}_{\mathrm{A}}\right)\right),
$$

The conversion $\mathrm{C}_{\mathrm{HPLC}}$ used in the above equation was calculated as $\mathbf{C}_{\mathrm{HPLC}}=\mathbf{e e}_{\mathrm{A}} /\left(\mathbf{e e}_{\mathrm{E}}+\mathbf{e} \mathbf{e}_{\mathrm{A}}\right)$, where $\mathbf{e e}_{\mathbf{E}}$ is the enantiomeric excess of the ester and $\mathbf{e e}_{\mathbf{A}}$ is the enantiomeric excess of the unreacted alcohol. The conversion values thus obtained were generally within $1-2 \%$ of the values obtained by NMR integration of crude reaction mixtures. 


\section{D) NMR study of DHIP.}

The following $0.2 \mathrm{M}$ stock solutions in $\mathrm{CDCl}_{3}$ were prepared: DHIP free base (see Section $\mathrm{B}$ ), acetic acid, acetic anhydride, and methanol. Using aliquots of these stock solutions, the following mixtures were prepared and their ${ }^{1} \mathrm{H}$ NMR spectra recorded (Figure S1).

a) $0.1 \mathrm{~mL} 0.2 \mathrm{M} \mathrm{DHIP}+0.5 \mathrm{~mL} \mathrm{CDCl}_{3}(0.033 \mathrm{M} \mathrm{DHIP}$ base $)$ - bright-yellow solution;

b) $0.1 \mathrm{~mL} 0.2 \mathrm{M} \mathrm{DHIP}+0.15 \mathrm{~mL} 0.2 \mathrm{M} \mathrm{AcOH}+0.35 \mathrm{~mL} \mathrm{CDCl}_{3}\left(0.033 \mathrm{M} \mathrm{H}^{-\mathrm{DHIP}}{ }^{+} \mathrm{AcO}^{-}\right)-$ the solution of DHIP faded somewhat upon addition of $\mathrm{AcOH}$;

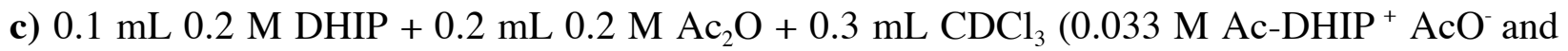
$0.033 \mathrm{M}$ unreacted $\mathrm{Ac}_{2} \mathrm{O}$ ) - the solution faded almost completely upon addition of 2 equivalents of $\mathrm{Ac}_{2} \mathrm{O}$; Only the acylated form of DHIP was observed by NMR.

d) $0.1 \mathrm{~mL} \mathrm{0.2} \mathrm{M} \mathrm{DHIP} \mathrm{+} \mathrm{0.2} \mathrm{mL} \mathrm{0.2} \mathrm{M} \mathrm{Ac} 2 \mathrm{O}+0.3 \mathrm{~mL} \mathrm{0.2} \mathrm{M} \mathrm{MeOH}\left(\left[\mathrm{Ac}_{2} \mathrm{O}\right]_{0}=0.066 \mathrm{M}\right.$; $\left.[\mathrm{MeOH}]_{0}=0.1 \mathrm{M} ;[\mathrm{DHIP}]_{0}=0.033 \mathrm{M}\right)$. After 40 minutes at room temperature, no $\mathrm{Ac}_{2} \mathrm{O}$

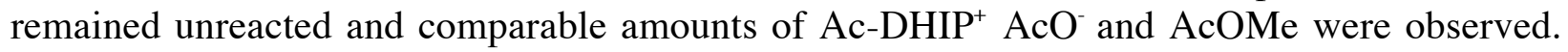
After 7 hours, the acyl transfer was essentially complete.

e) $0.1 \mathrm{~mL} \mathrm{CDCl}{ }_{3}+0.2 \mathrm{~mL} 0.2 \mathrm{M} \mathrm{Ac}_{2} \mathrm{O}+0.3 \mathrm{~mL} \mathrm{0.2} \mathrm{M} \mathrm{MeOH}\left(\left[\mathrm{Ac}_{2} \mathrm{O}\right]_{0}=0.066 \mathrm{M}\right.$; $[\mathrm{MeOH}]_{0}=$ $0.1 \mathrm{M}$ ) - No reaction between $\mathrm{Ac}_{2} \mathrm{O}$ and $\mathrm{MeOH}$ was observed in the absence of DHIP after 50 minutes.

\section{Figure S1.}

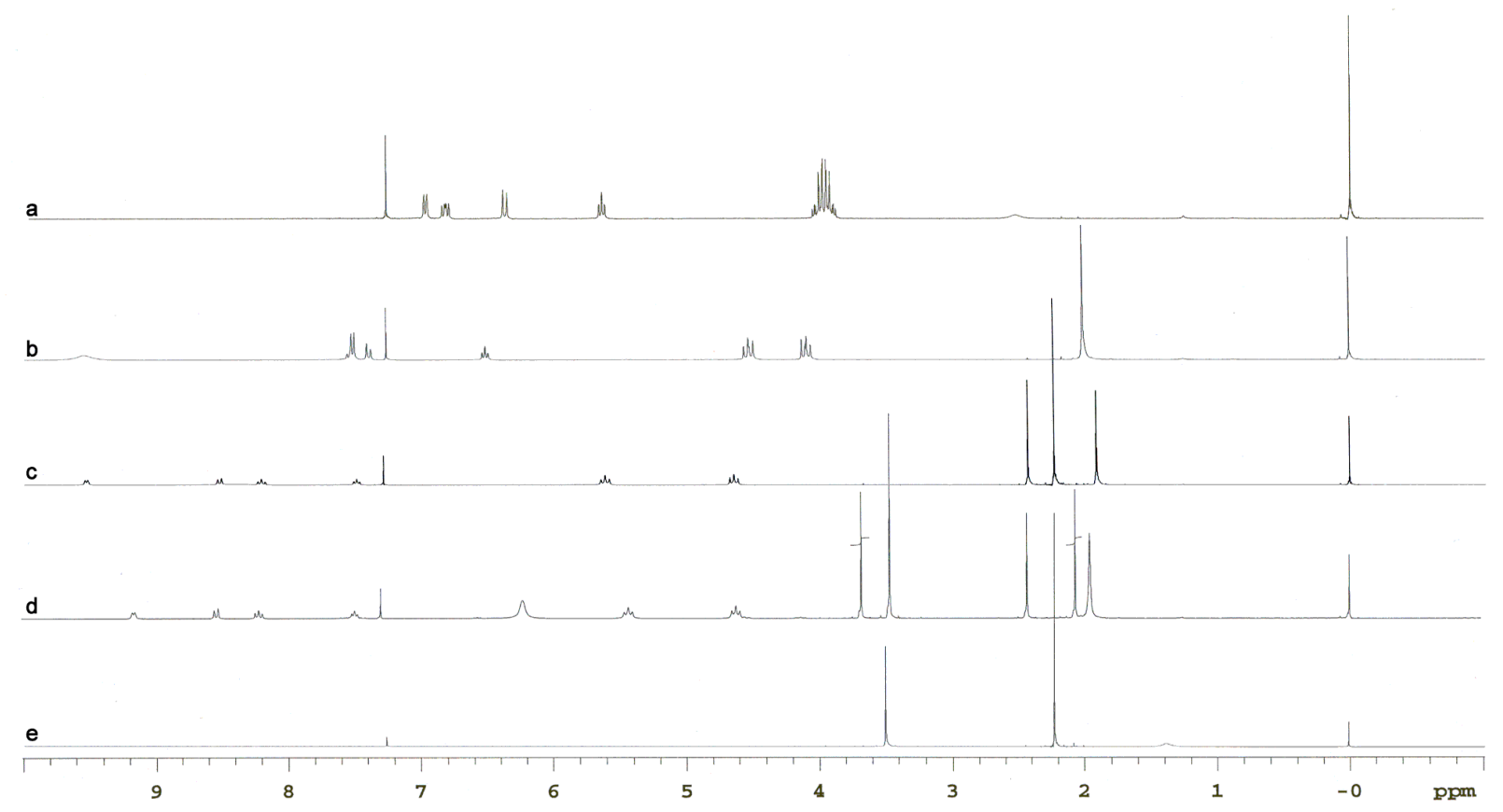




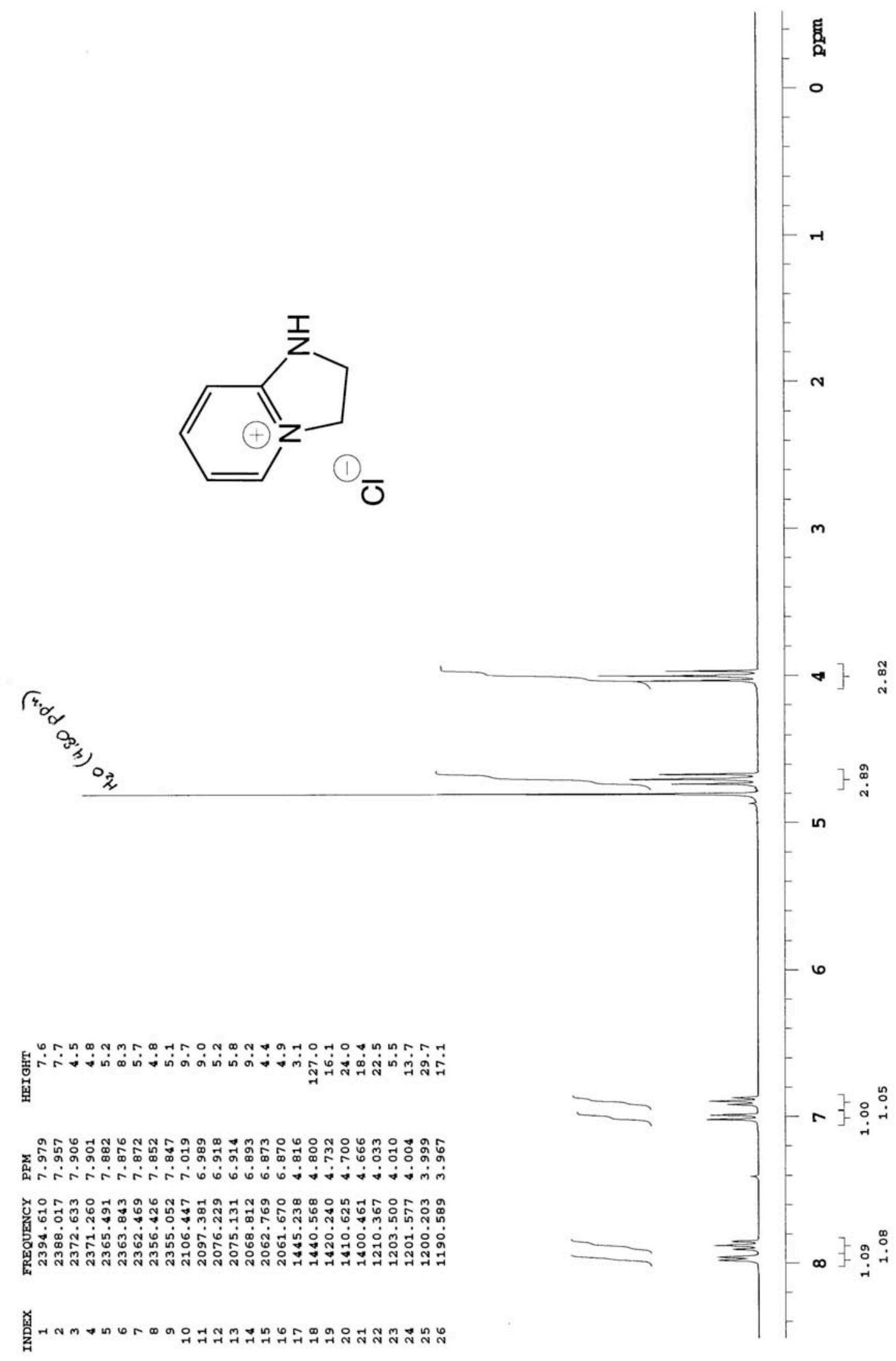



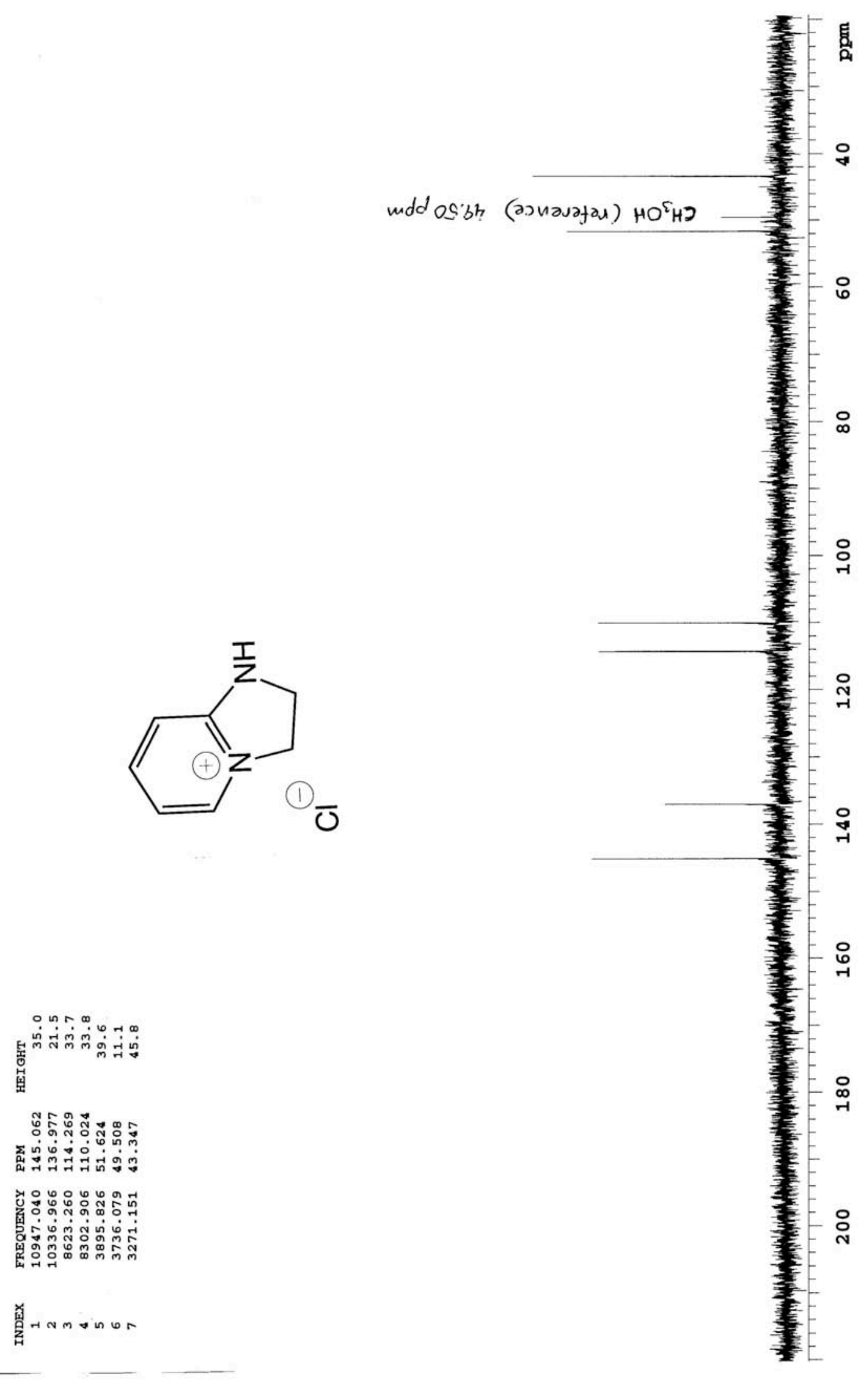

13 


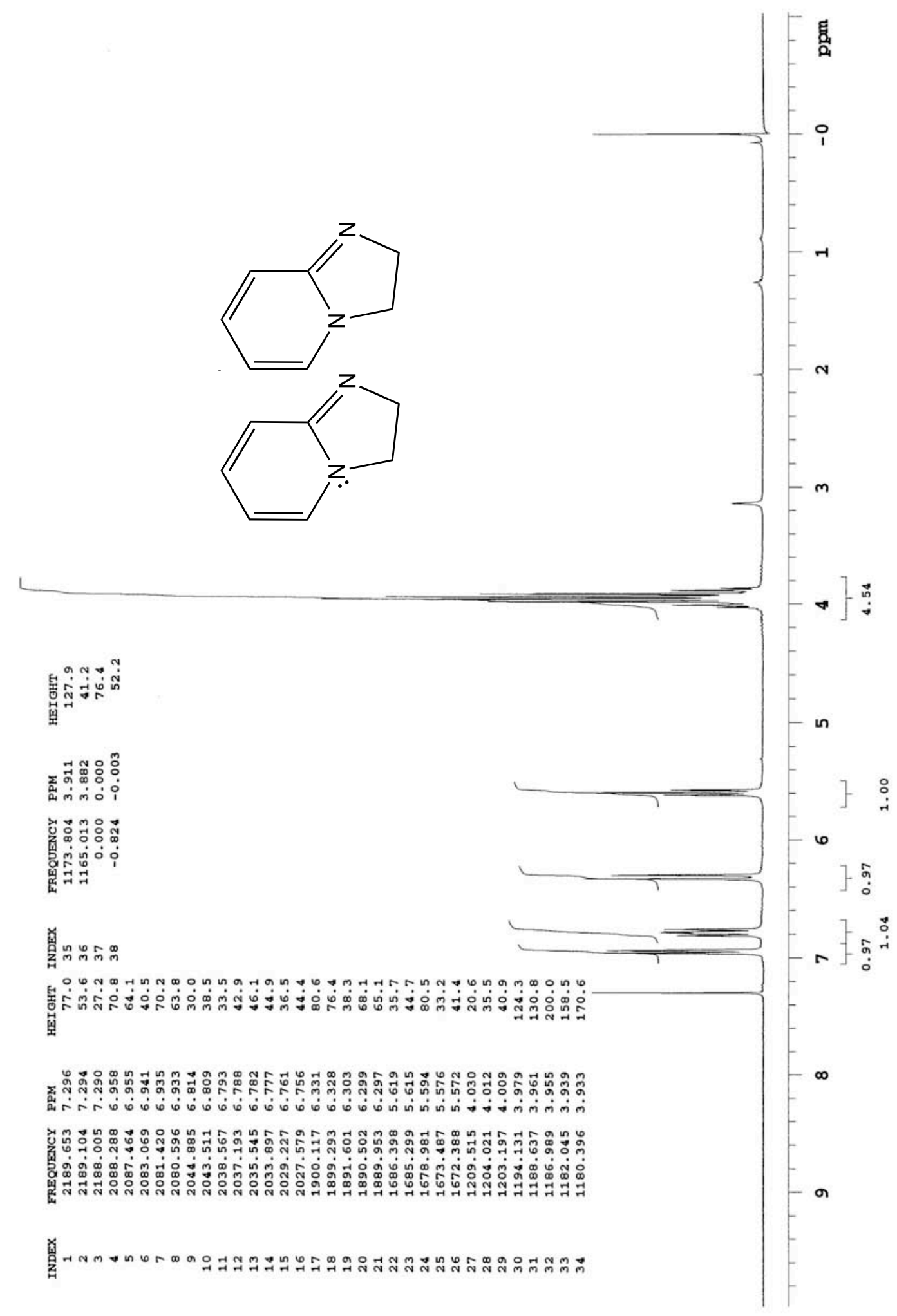



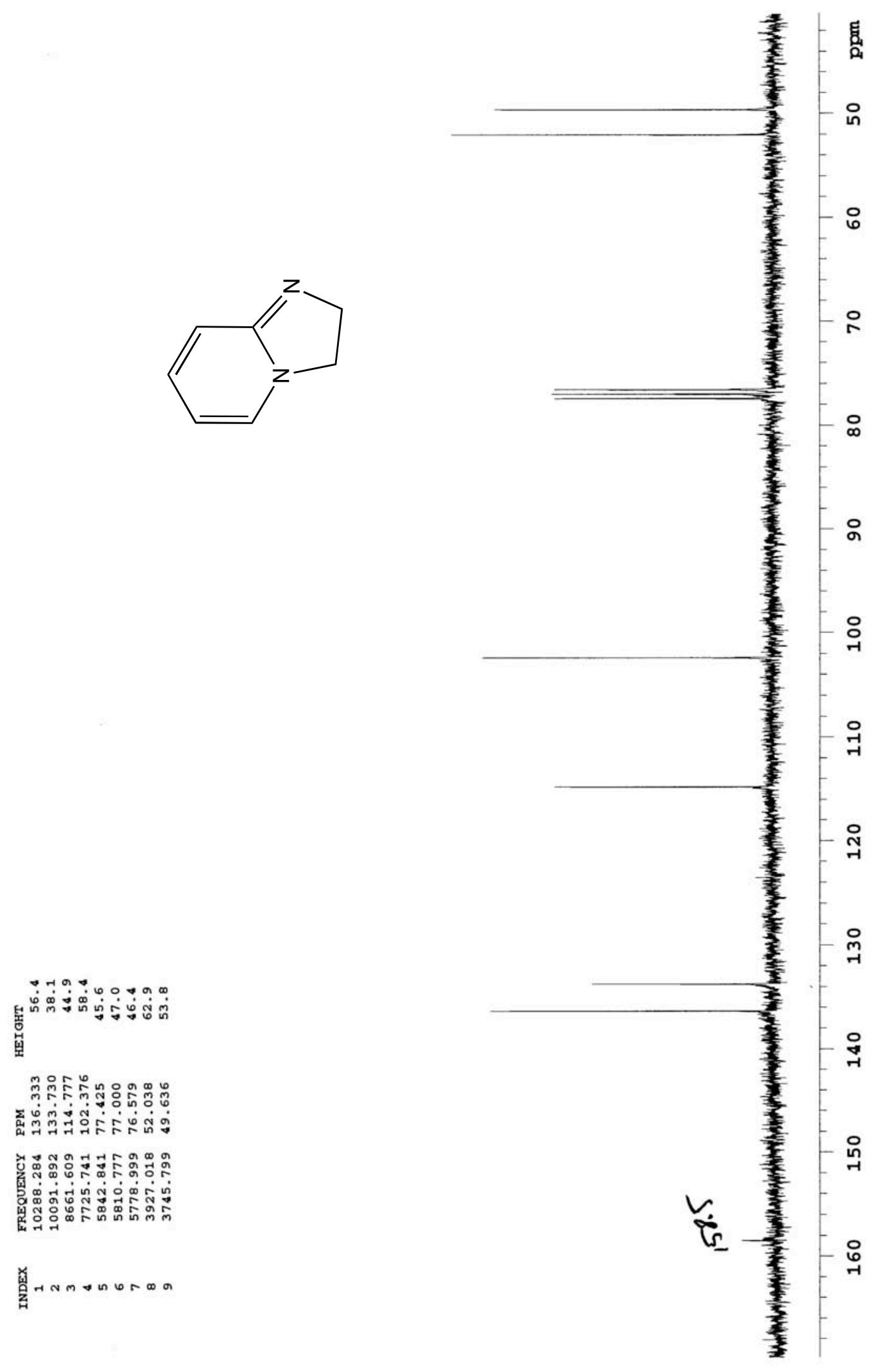


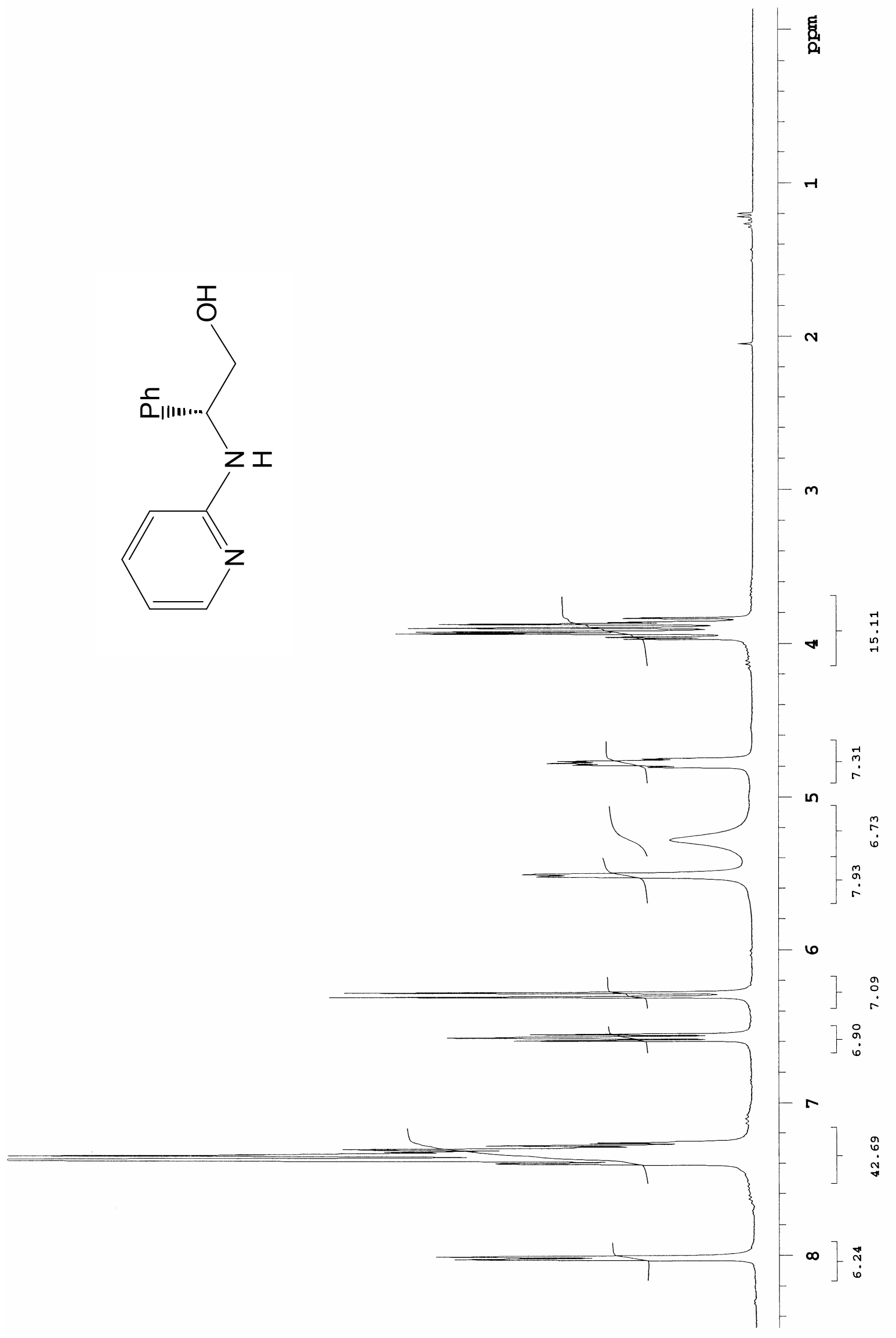




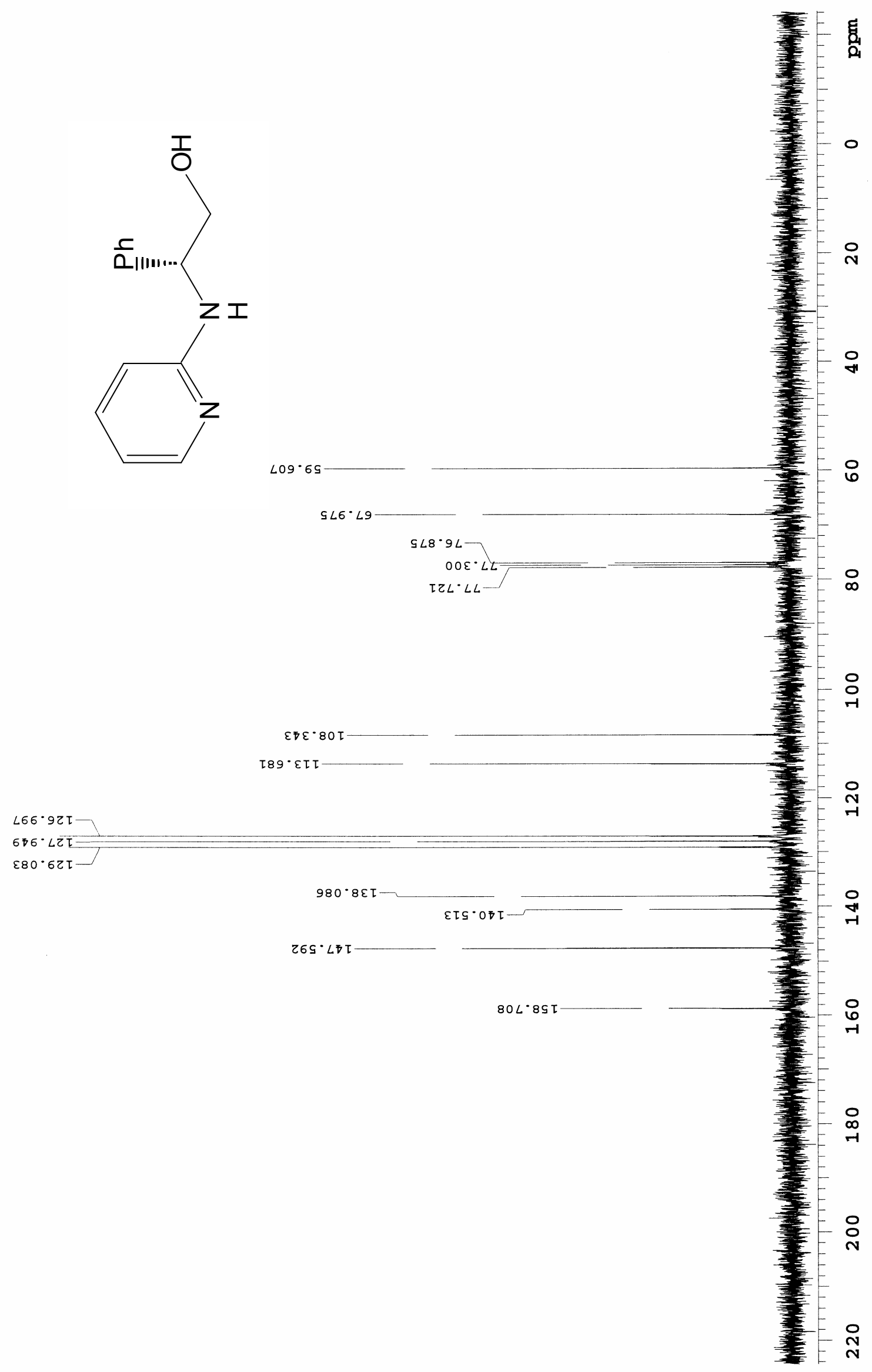




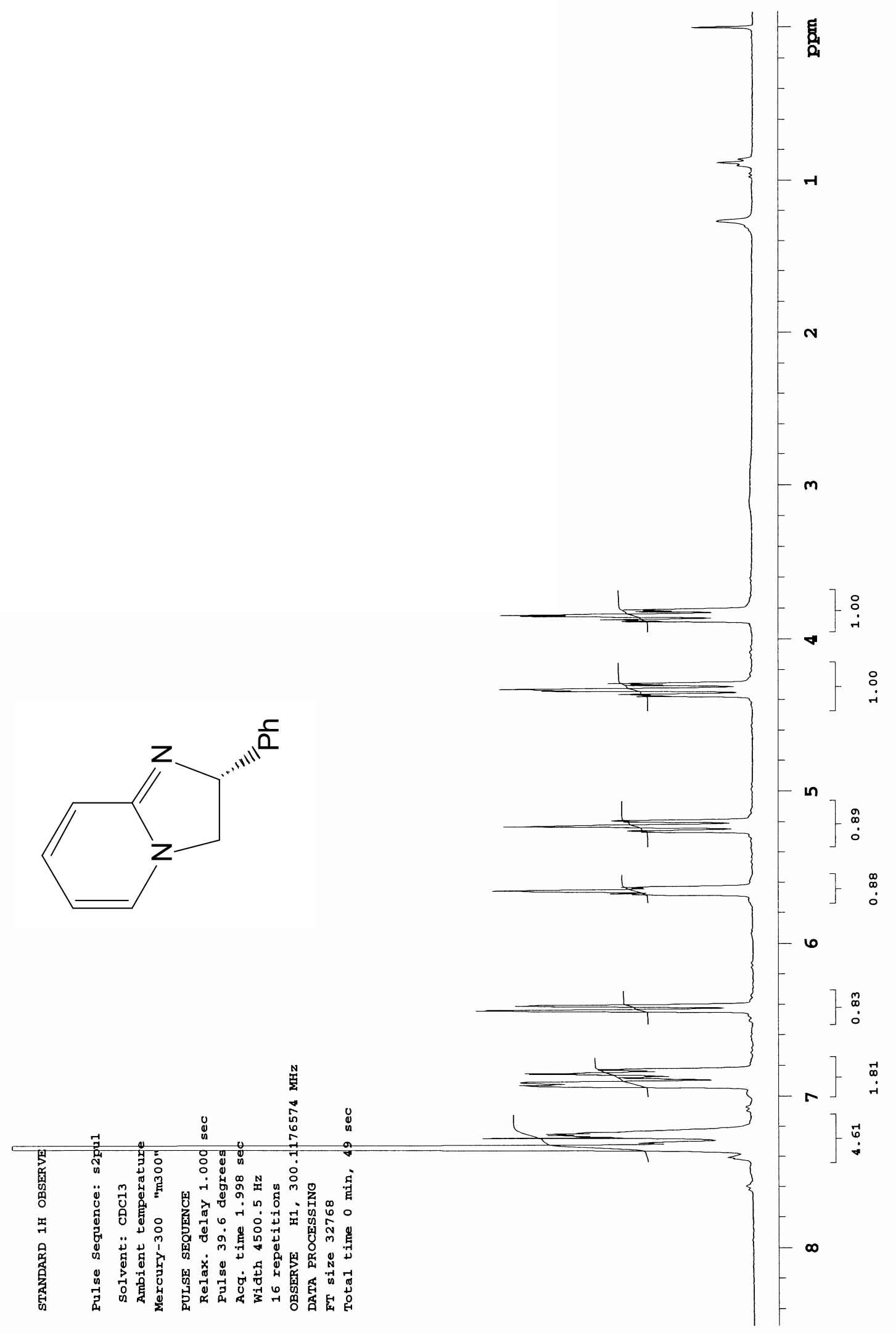




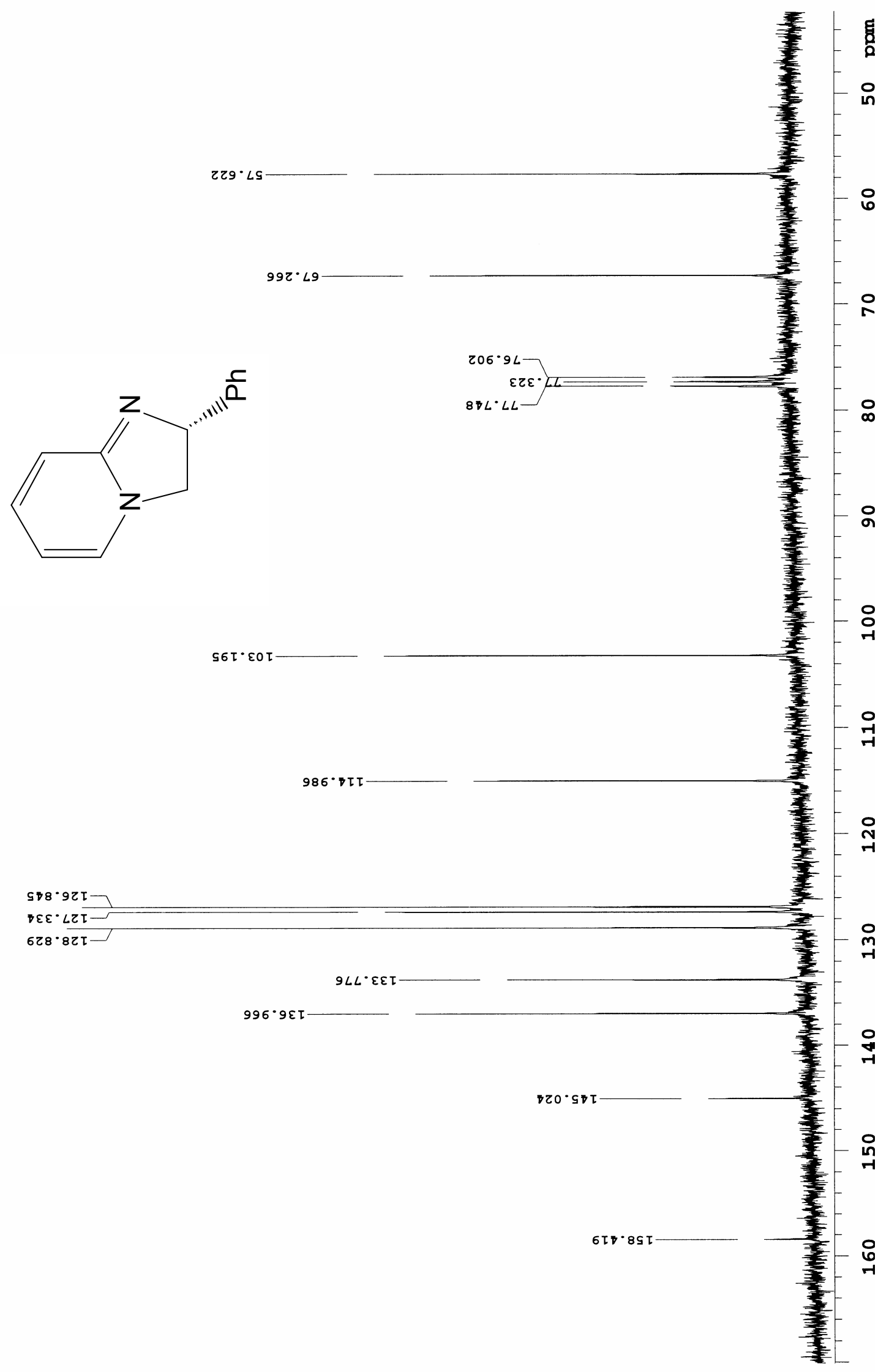




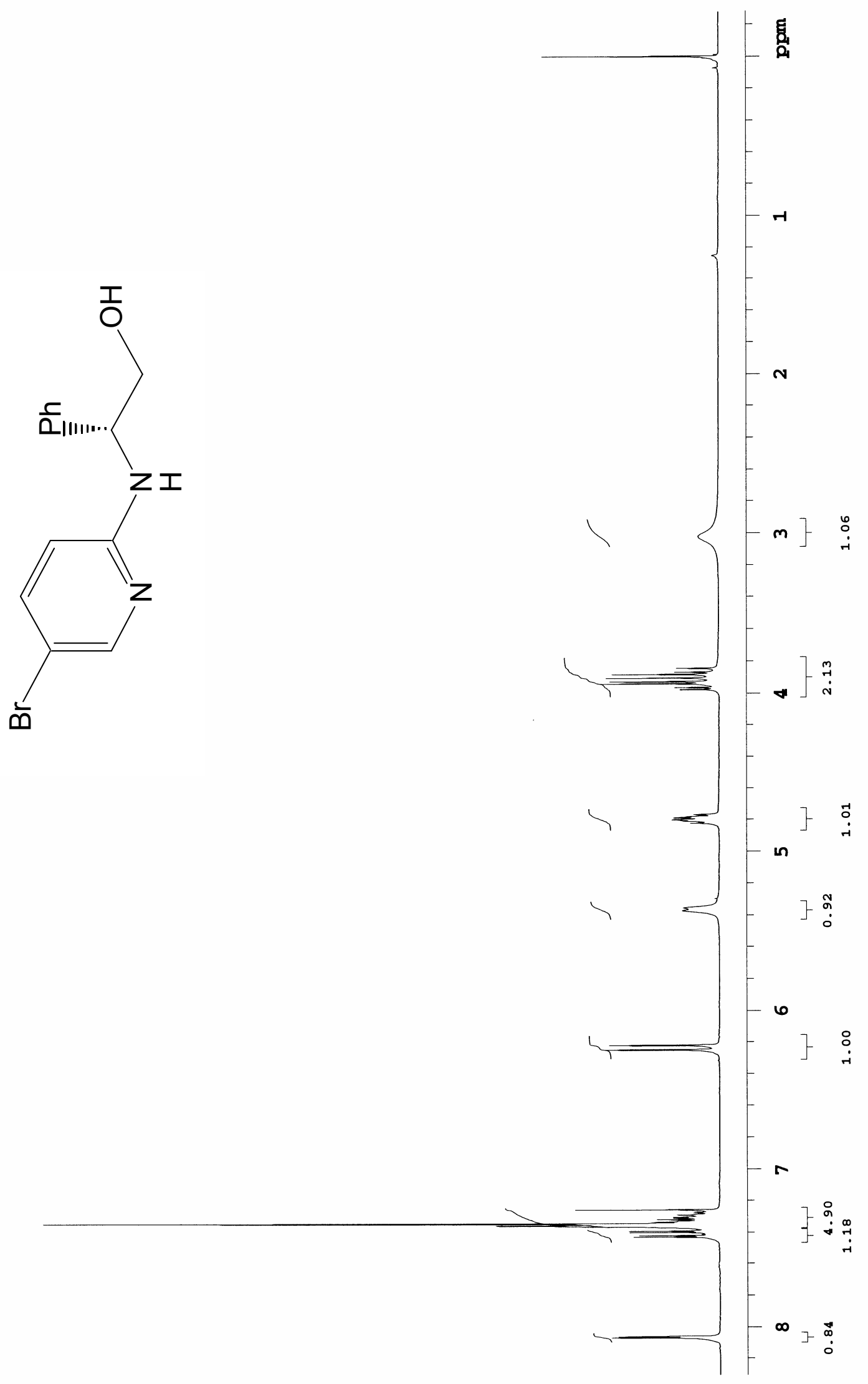




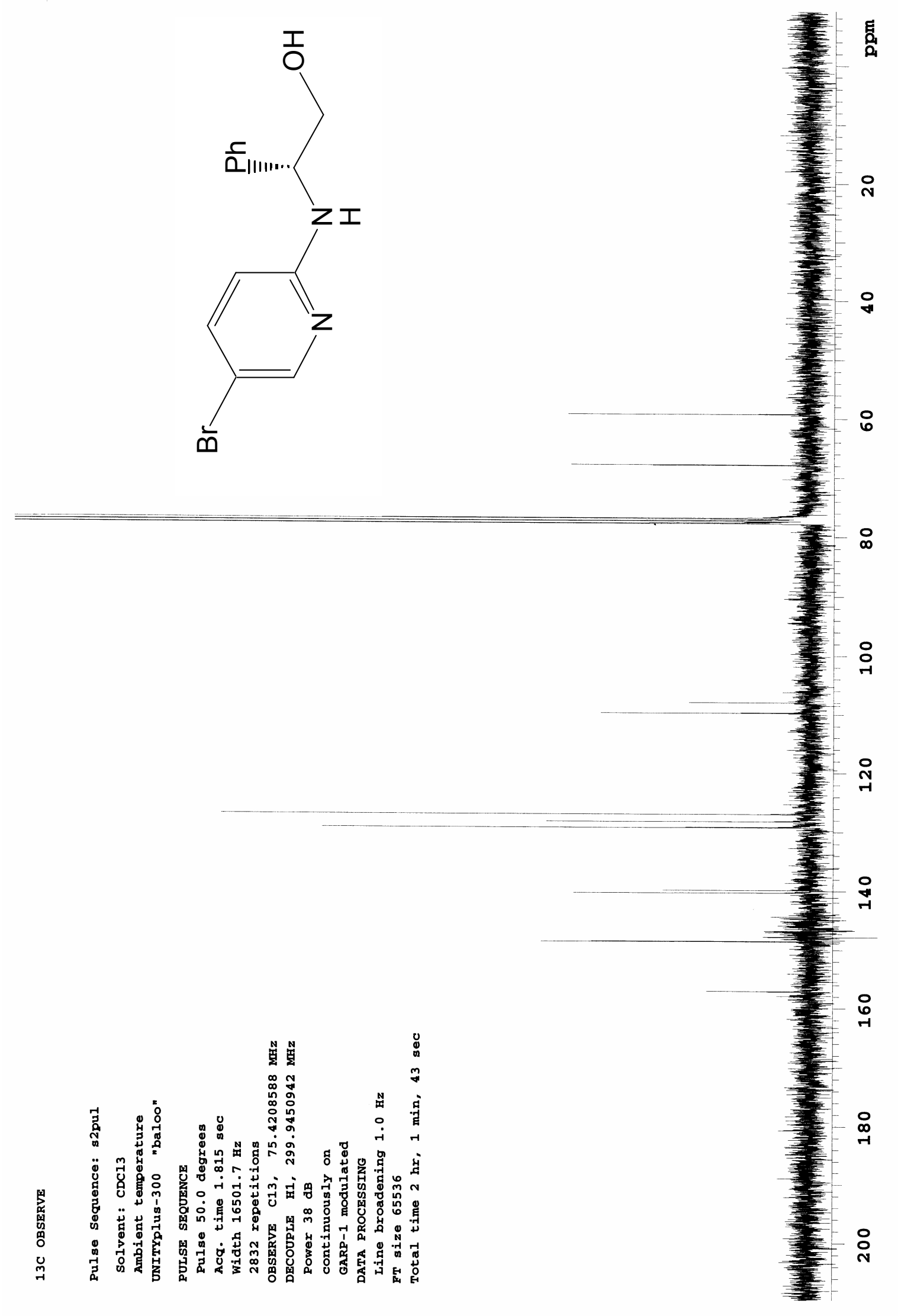




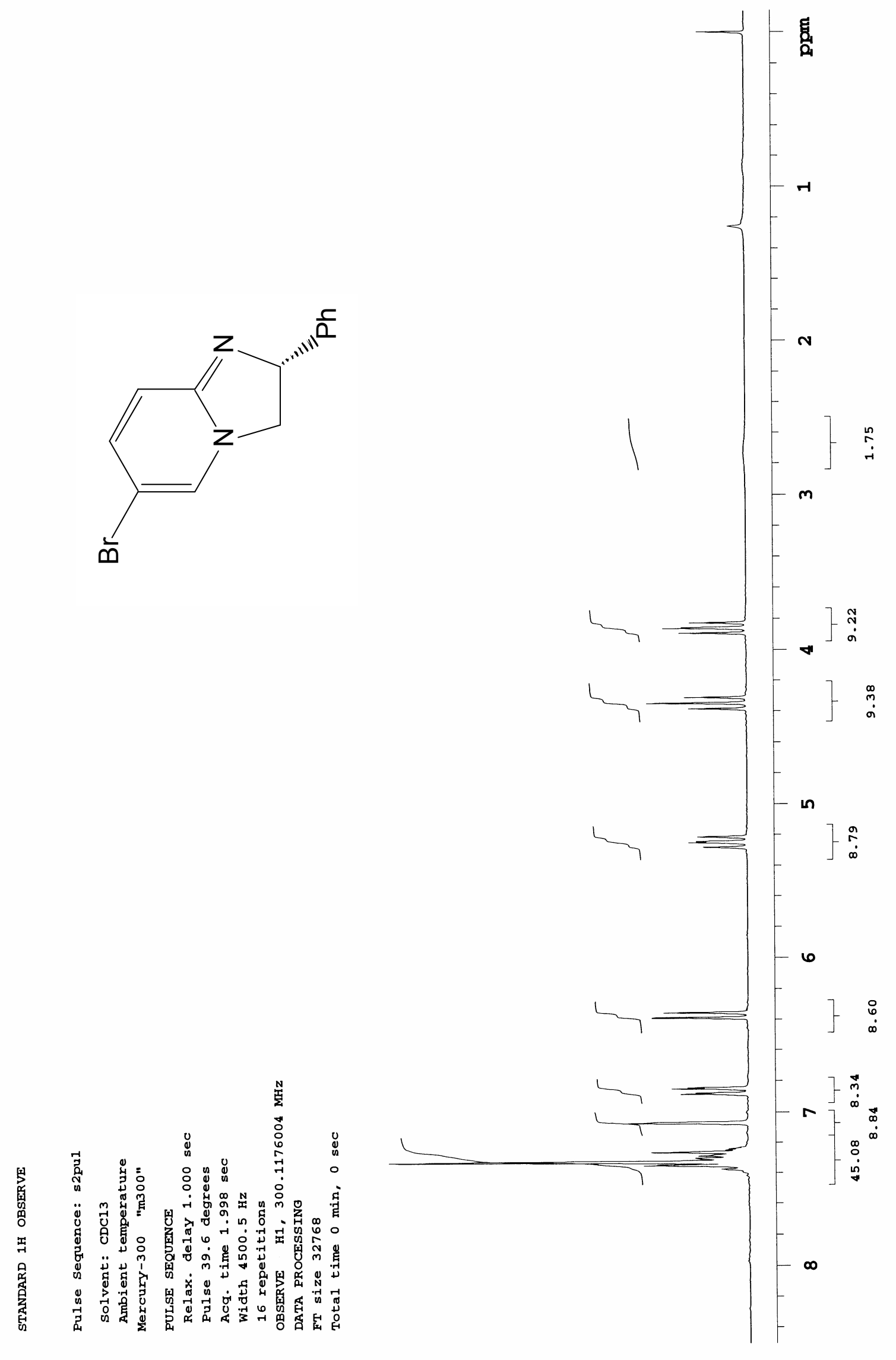




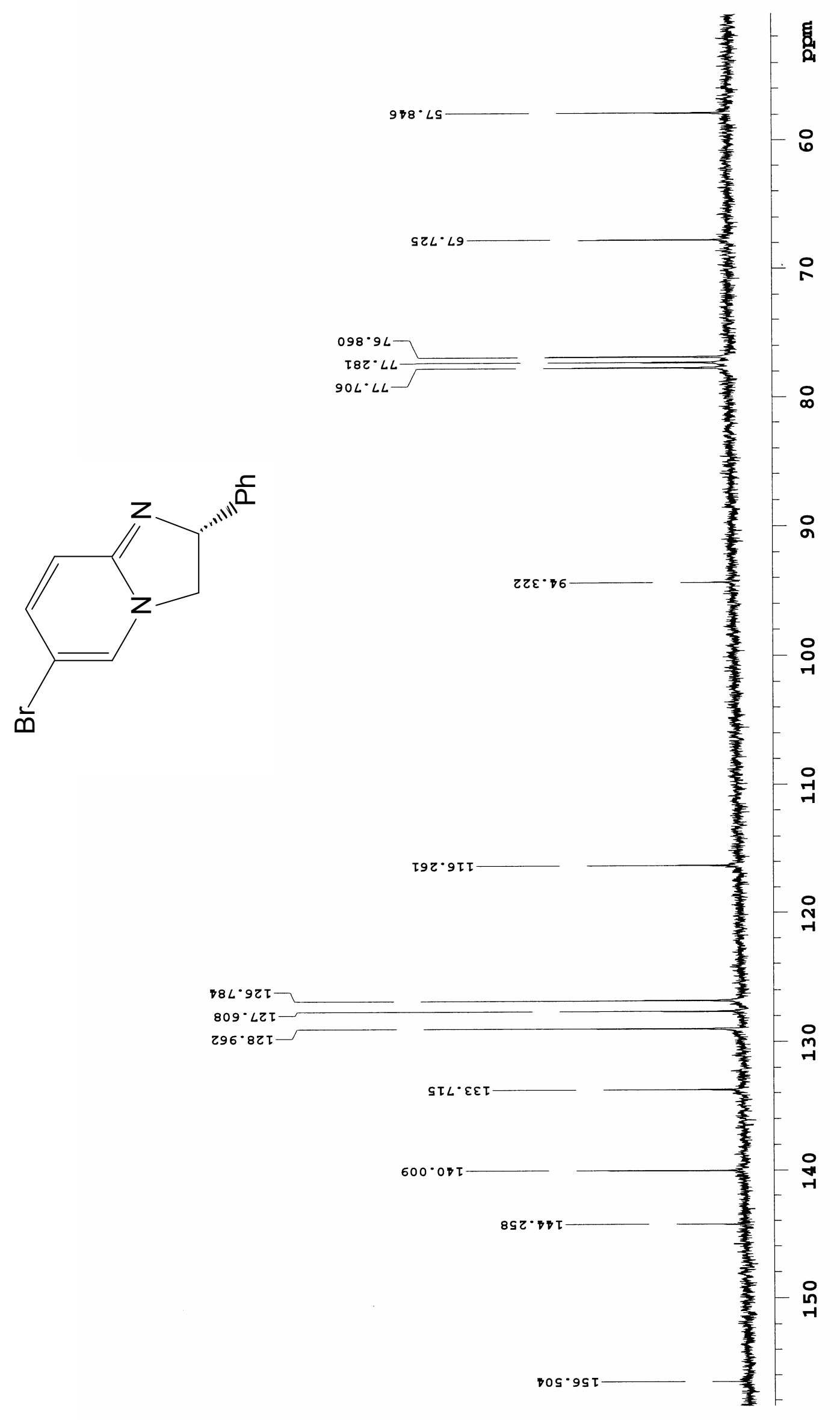




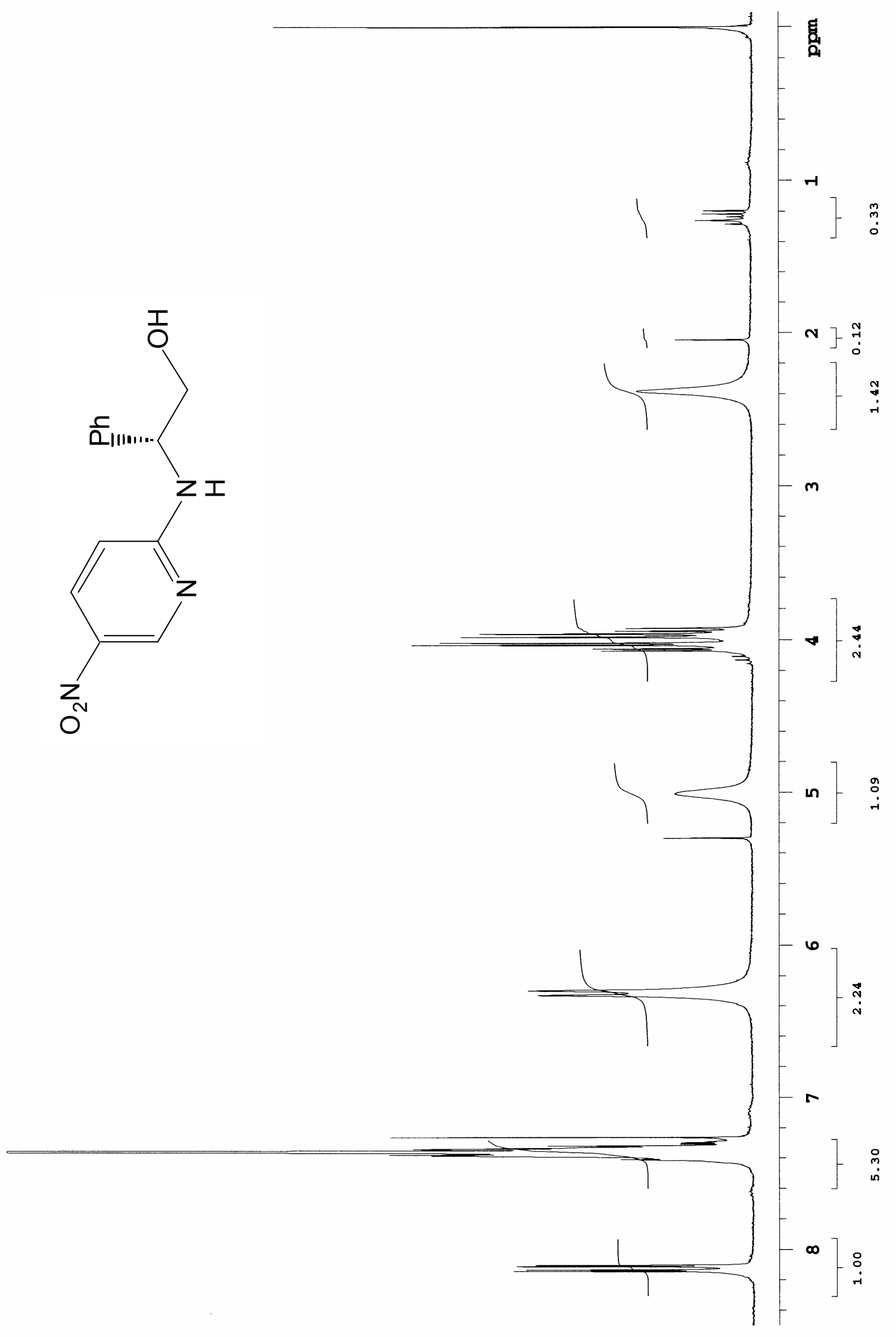




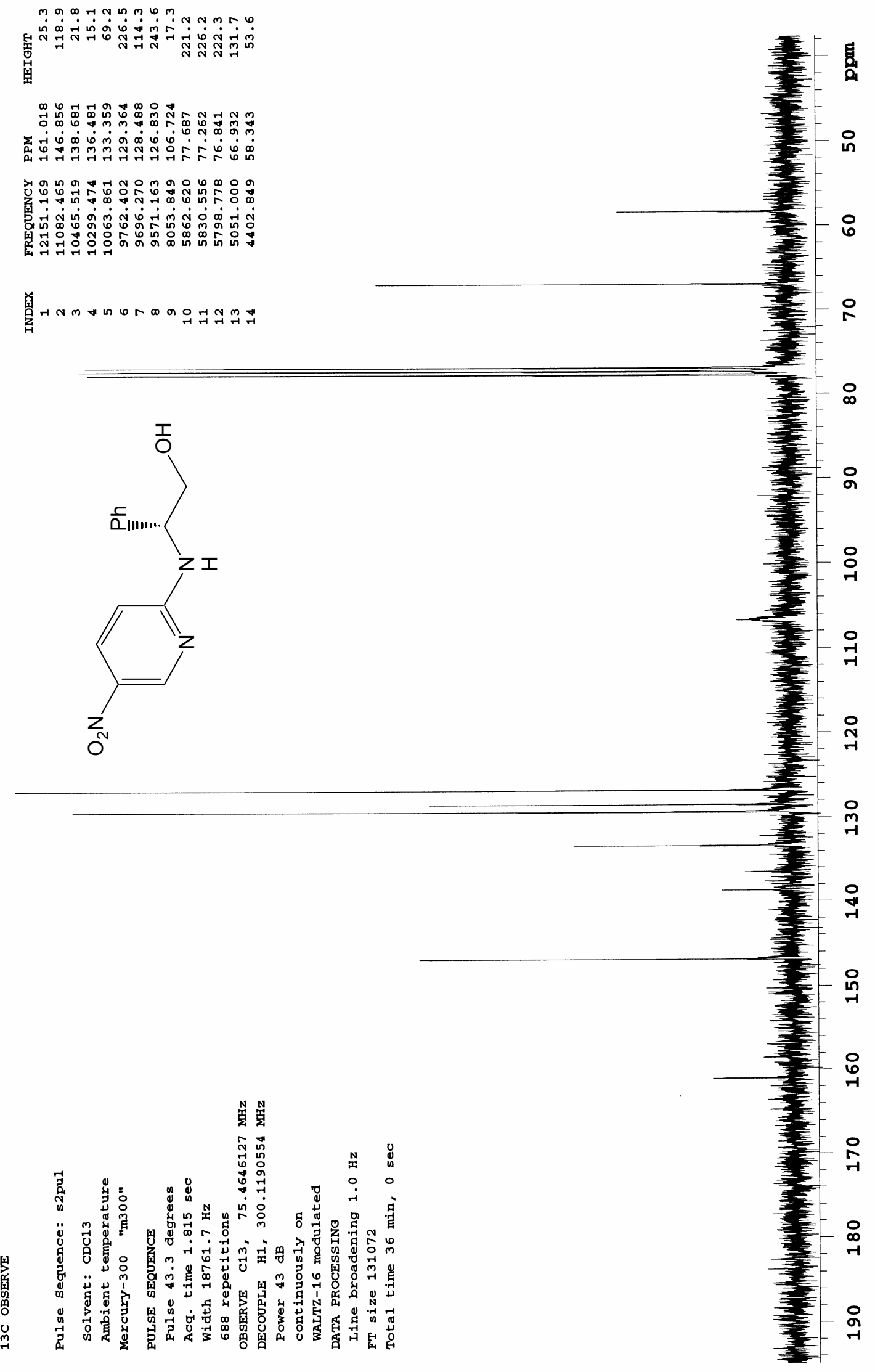




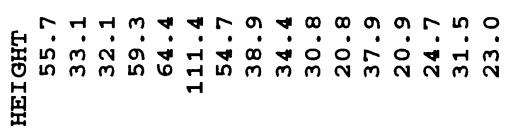

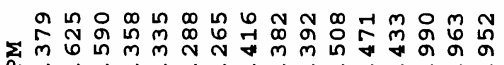

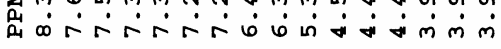

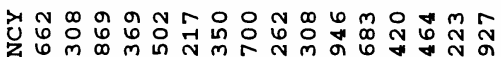

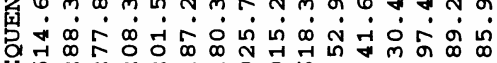

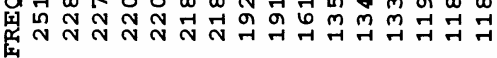

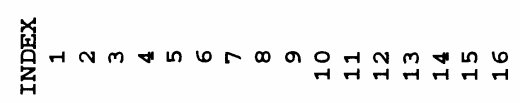
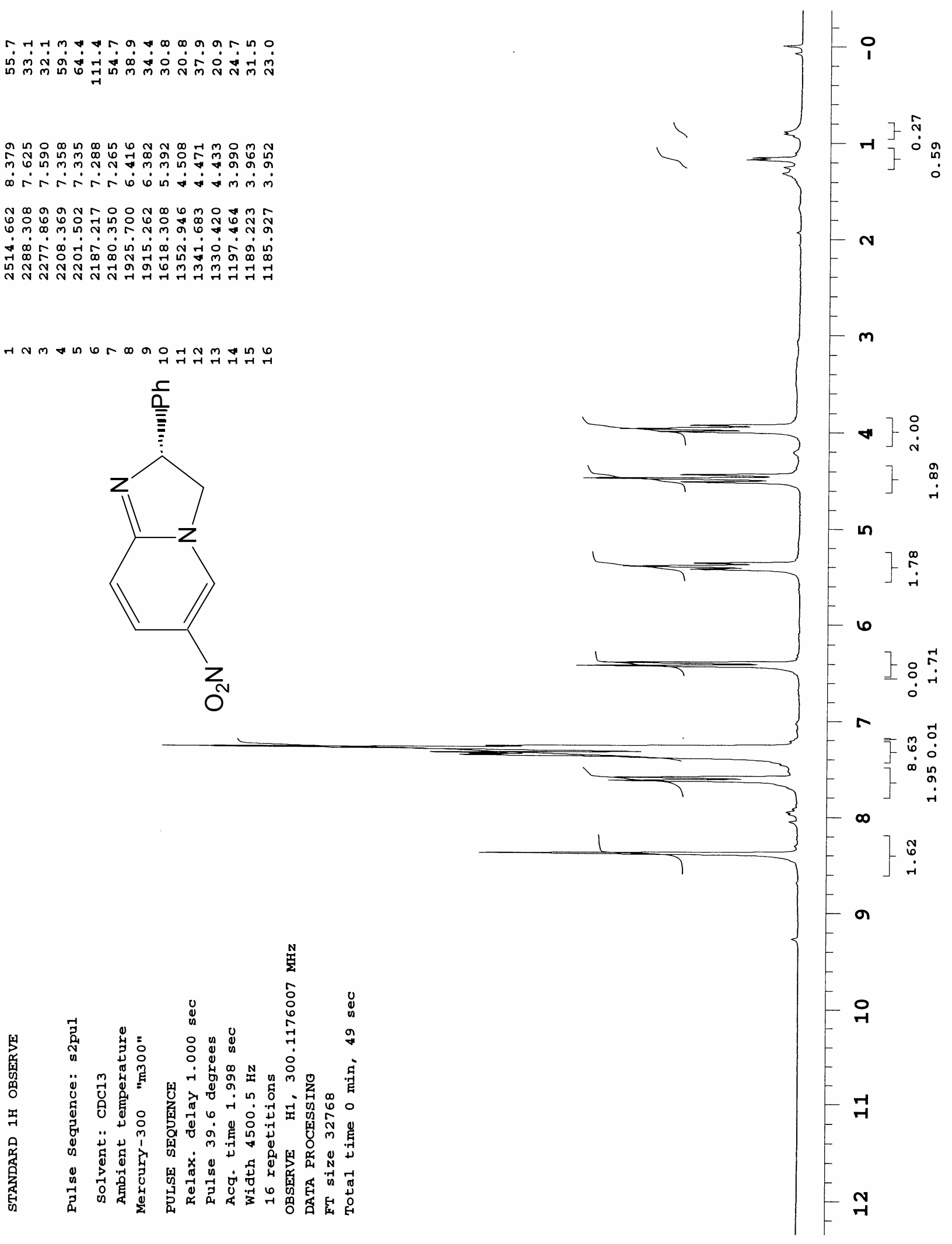

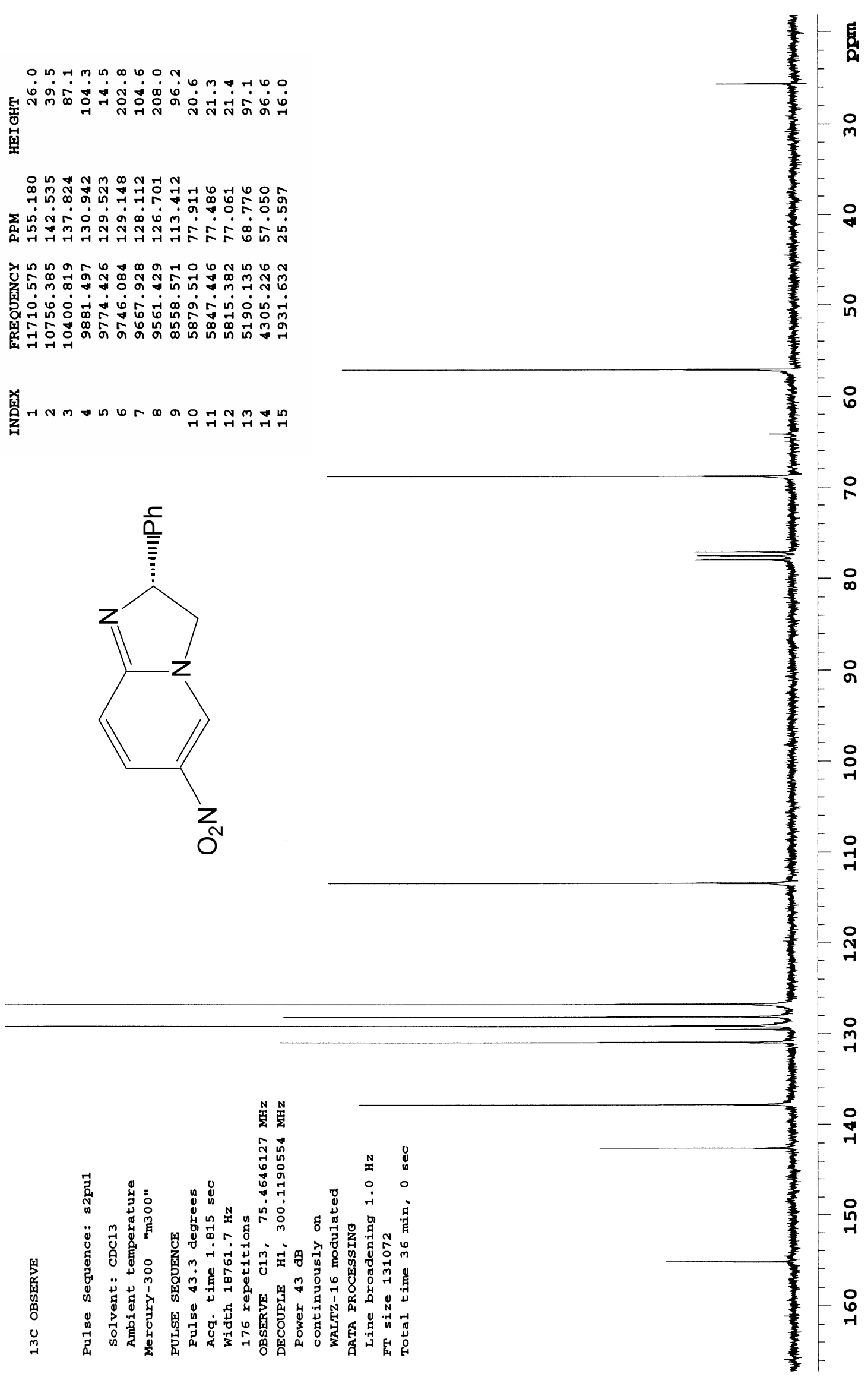


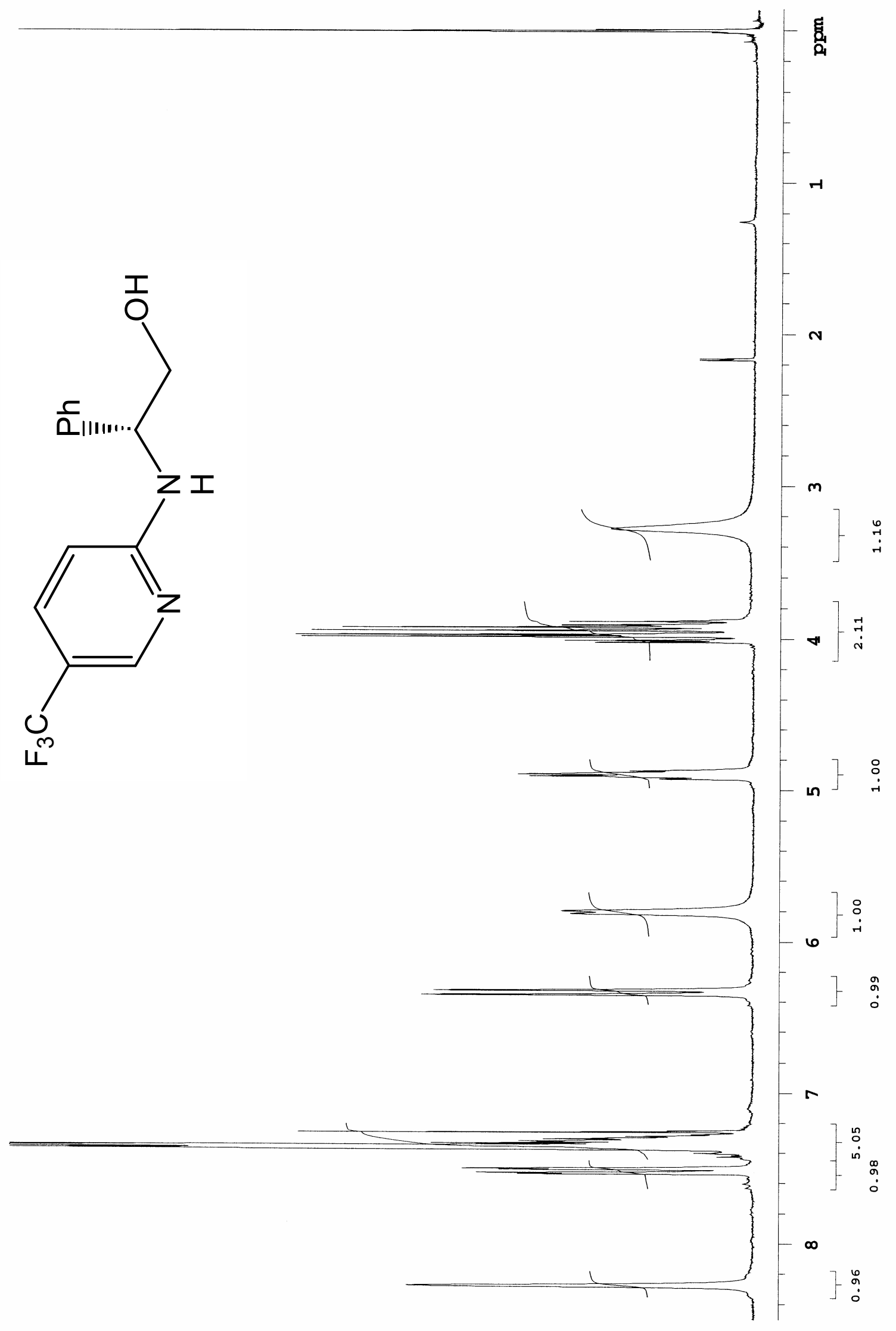




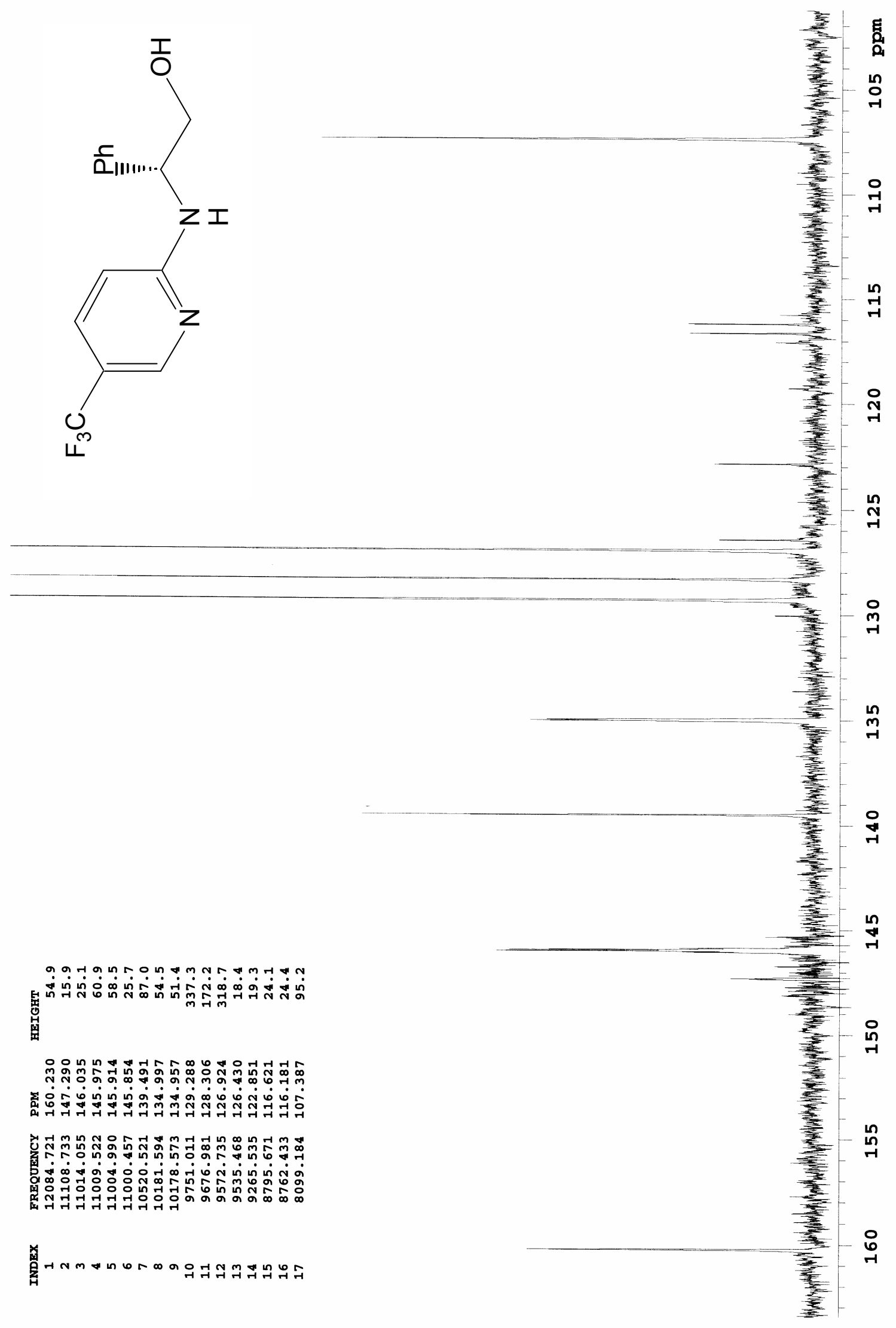




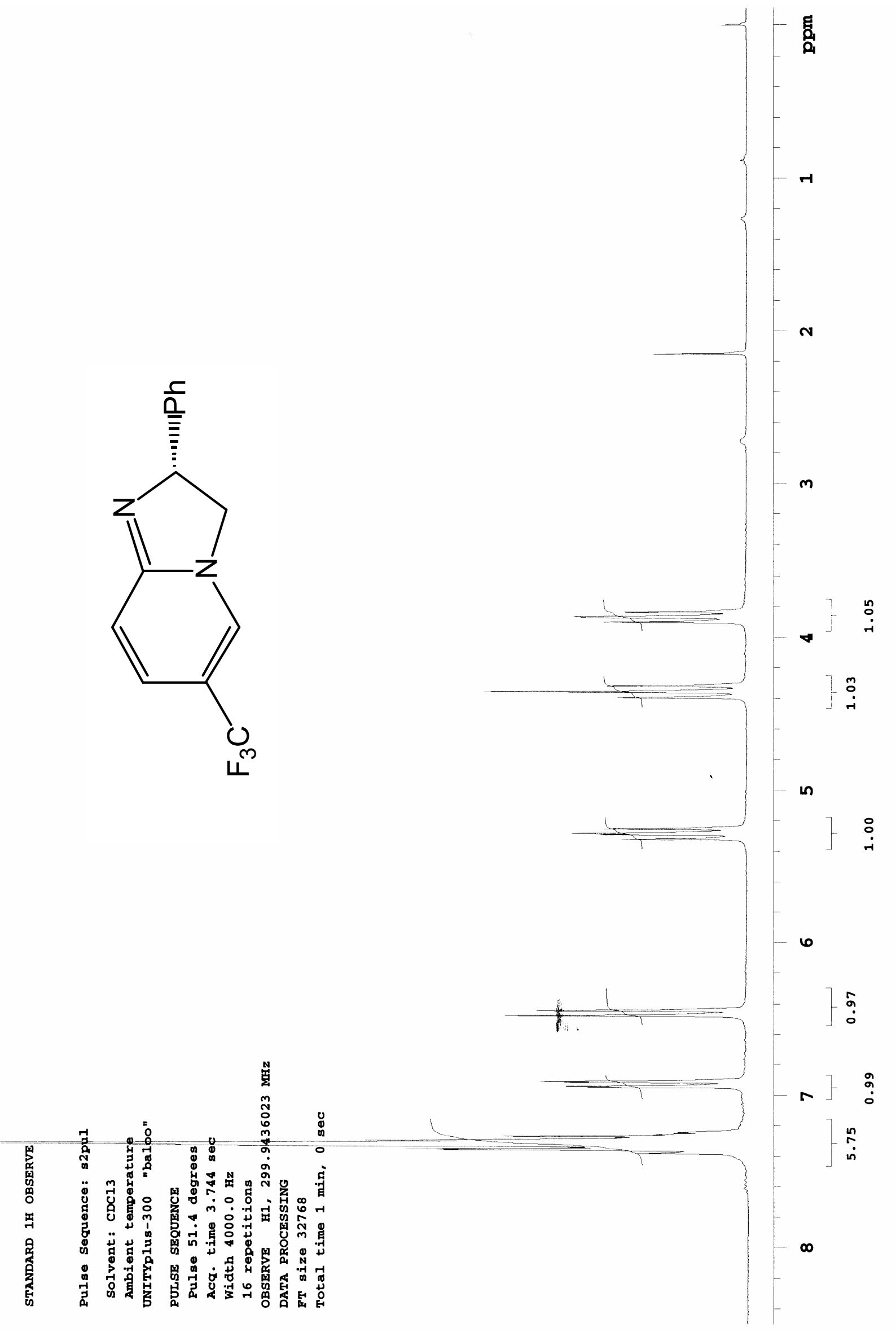



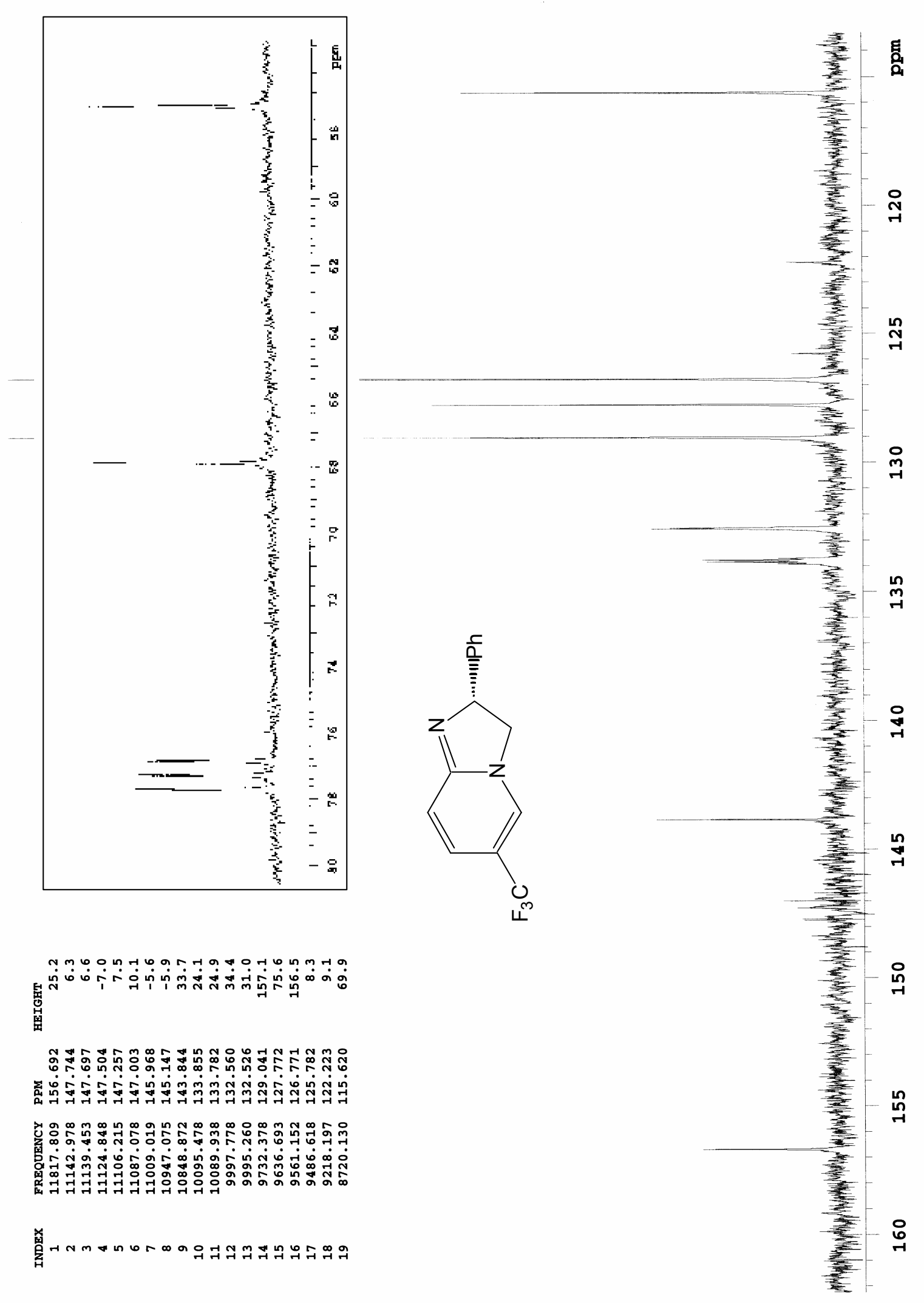

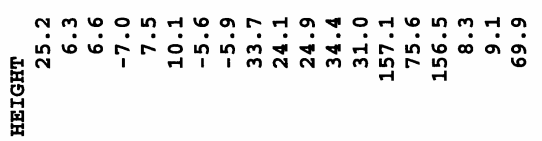

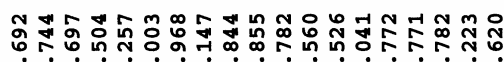

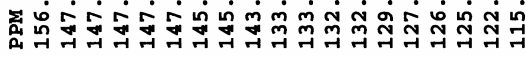

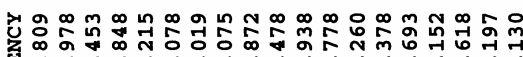

(1)

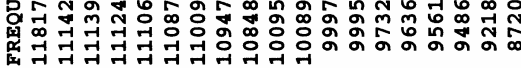

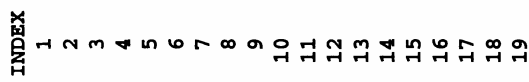

: 